\title{
Collateralized Networks
}

\author{
Samim Ghamami* Paul Glasserman ${ }^{\dagger} \quad$ H. Peyton Young ${ }^{\ddagger}$
}

June 2019; this version November 2020

\begin{abstract}
This paper studies the spread of losses and defaults in financial networks with two interrelated features: collateral requirements and alternative contract termination rules. When collateral is committed to a firm's counterparties, a solvent firm may default if it lacks sufficient liquid assets to meet its payment obligations. Collateral requirements can thus increase defaults and payment shortfalls. Moreover, one firm may benefit from the failure of another if the failure frees collateral committed by the surviving firm, giving it additional resources to make other payments. Contract termination at default may also improve the ability of other firms to meet their obligations through access to collateral. As a consequence of these features, the timing of payments and collateral liquidation must be carefully specified to establish the existence of payments that clear the network. Using this framework, we show that dedicated collateral may lead to more defaults than pooled collateral; we study the consequences of illiquid collateral for the spread of losses through fire sales; we compare networks with and without selective contract termination; and we analyze the impact of alternative resolution and bankruptcy stay rules that limit the seizure of collateral at default. Under an upper bound on derivatives leverage, full termination reduces payment shortfalls compared with selective termination.
\end{abstract}

JEL Classifications: D53, D70, E44, G1, G28, G33, K22

Keywords: Contagion, OTC markets, financial regulation, network, fire sales, collateral, automatic stays for qualified financial contracts

\section{Introduction}

This paper studies the spread of losses and defaults through financial networks when payment obligations are at least partly secured by collateral. A combination of changes in regulation and industry practices following the financial crisis of 2007-2009 have greatly expanded the use of collateral in trading and lending. In the over-the-counter (OTC) derivatives market, most

\footnotetext{
*University of California, Berkeley, New York University, and the Financial Services Forum, email: samim_ghamami@berkeley.edu. Ghamami carried out the work on an earlier version of this paper at Goldman Sachs before moving to FSF through Goldman Sachs. The views in this paper do not necessarily reflect those of the FSF, Goldman Sachs, and other U.S. Global Systemically Important Banks.

${ }^{\dagger}$ Columbia Business School, email: pg20@columbia.edu.

${ }^{\ddagger}$ University of Oxford, email: peyton.young@economics.ox.ac.uk.
} 
standardized contracts now trade through central counterparties, which require participants to post collateral in the form of initial margin (IM). The part of the market that continues to trade bilaterally is now also subject to IM requirements. Similarly, unsecured interbank lending is far lower than its pre-crisis levels and has mostly been replaced by collateralized lending through repurchase agreements. See, e.g., Duffie [13, Ghamami and Glasserman [24], Financial Stability Board [21], and pages 107-126 of the U.S. Department of Treasury Report [43] for background.

Collateral provides a buffer against the spread of losses: if one party to a contract defaults on a payment obligation, its counterparty can seize available collateral to offset the loss. In this sense, collateral supports financial stability.

But, as our analysis will show, this is not the whole story. We highlight additional mechanisms that complicate the impact of collateral. First, committing collateral to specific contracts and counterparties can lead to an ex post inefficient allocation of a firm's assets: a firm may find itself unable to make a current payment obligation on one contract despite having posted collateral to protect future potential obligations on other contracts. Firms do not ordinarily have the option to terminate contracts to recover posted collateral. In reducing counterparty credit risk, collateral requirements can increase strains on funding liquidity, because collateral requirements create additional funding needs 11 These effects are intimately connected to contract termination rights, because terminating a contract can provide access to collateral and, indeed, is ordinarily necessary for access to collateral.

Collateral held in less liquid assets creates a further consequence for contagion. At the failure of one institution, its counterparties would seize and liquidate collateral. This sell-off or fire sale could drive down the price of the collateral assets, creating market losses at other firms holding those assets. In particular, firms that had posted similar assets as collateral would find themselves with a collateral shortfall — and an obligation to add collateral — as a result of the fire sale. See Stein [40], Shleifer and Vishny [36], Chapters 1 and 2 of Gennaioli and Shleifer [23], and the references therein for background on the role of fire sales in financial crises.

We develop these ideas in a network model. The nodes of the network are parties to financial contracts; for brevity, we sometimes refer to these as banks, though we have in mind a broader set of financial and even non-financial companies. The nodes are linked through contracts that carry payment obligations. We take the network configuration as an input to our analysis; our model does not seek to explain how a particular configuration comes about.

We build on the standard framework of Eisenberg and Noe [17]. The Eisenberg-Noe model

\footnotetext{
${ }^{1}$ Researchers have also debated the implications for economic growth of the demand for safe assets as collateral; see Duffie et al. 14] and Sidanius and Zikes [37. for estimates of the demand. Our analysis addresses the distribution and allocation of collateral, rather than its overall level.
} 
takes a set of nodes with balance sheets linked through uncollateralized payment obligations and identifies one or more clearing vectors. A clearing vector describes a set of actual payments under which a node never pays more than it owes, all contracts have equal seniority, and nodes face limited liability. These properties lead to a fixed-point characterization of clearing payments. The Eisenberg-Noe model has been extended to cover many other features, including bankruptcy costs (Rogers and Veraart [35]) and claims of different seniority (Elsinger [18]) or maturity (Kusnetsov and Veraart [32]); see Cabrales, Gale, and Gottardi [8], Glasserman and Young [25], Hurd [28, and Jackson and Pernoud [30] for overviews and extensive references. Despite these many extensions, the inclusion of collateral poses special complications and requires a departure from the usual solution approach.

In a collateralized network, the failure of one node may improve the ability of other nodes to meet their obligations. If a surviving node had committed collateral to a contract with the failed node, the failure frees that collateral, providing the surviving node additional resources to make other payments. Indeed, the freed collateral might even be necessary for the surviving node to meet its obligations, in which case the failure of one node prevents the failure of another. We will show that under these circumstances the notion of clearing payments may not be well-defined.

In an equilibrium of the Eisenberg-Noe model, the timing of events (payments and defaults) is immaterial, and these events may be understood as occurring simultaneously. In modeling collateral, we separate the timing of two types of events following a default. We assume that creditors have immediate access to collateral posted by the defaulting node; but the freeing of collateral posted to the default node will follow a short delay. This modeling choice is supported by Key Principle 5 of the BCBS-IOSCO [4] (p.20) principles on margin requirements for noncleared derivatives: Initial margin collected should be held in such a way as to ensure that (i) the margin collected is immediately available to the collecting party in the event of the counterparty's default, and (ii) the collected margin must be subject to arrangements that protect the posting party to the extent possible under applicable law in the event that the collecting party enters bankruptcy. The return of collateral in (ii) does not carry the same urgency as the access to collateral in (i), so we will not assume they occur simultaneously.

By separating these events we show that we can arrive at a well-defined set of clearing payments. The separation eliminates the possibility that the failure of one node could prevent the failure of another node by freeing collateral - a scenario we see as unrealistic as well as a complication for the analysis. Along with the clearing payments, we characterize the set of defaulting nodes and the redistribution of collateral. 
We use this framework to evaluate the effects on a network of various policy options related to collateral and contract termination rights, because contract termination controls access to collateral. We take as our measures of financial stability the size of the default set and the network's total payment shortfall. We examine these measures in various scenarios and establish the following conclusions.

(i) Costless termination. As a starting point for comparion, we consider networks in which nodes are free to terminate contracts, and we show that under this assumption posting collateral is equivalent to making certain payments, so collateral plays no essential role. This reference point establishes the close connection between access to collateral and restrictions on contract termination that runs through the rest of our analysis.

(ii) Pooled collateral. We investigate the tradeoff between committing collateral to specific counterparties versus pooling collateral and holding additional cash. This tradeoff is analogous to the comparison between collateral requirements and capital requirements - capital absorbs any type of loss, whereas collateral protects specific obligations. Although pooling may appear to allow a better allocation of resources, we show that pooling is guaranteed to reduce defaults under additional conditions. For instance, we show that when committed collateral exceeds current payment obligations, pooling reduces defaults and does not affect payment shortfalls. This result is applicable with derivative contracts. Since in OTC derivatives markets, collateral in the form of initial margin captures in part extreme potential future exposures, it can exceed current payments (Ghamami [27]). In general, however, pooling may produce larger or smaller payment shortfalls, depending on a node's position in the network, so the tradeoff cannot be resolved by considering a node in isolation.

(iii) Collateral illiquidity. We expand our model to capture the potential spread of losses through collateral fire sales. We extend the method of Cifuentes, Ferrucci, and Shin [10], which models illiquidity through a price-impact function. When a node fails, its creditors liquidate collateral, driving down its price and lowering the value of similar assets held by other nodes. This price-mediated channel amplifies losses beyond the direct effect of missed payments. Collateral illiquidity increases defaults and payment shortfalls.

(iv) Automatic stays. We consider the effect of a stay under which payments are made before collateral is liquidated and show that it has no effect on defaults or payment shortfalls when collateral is liquid. We interpret this point as consistent with the policy recommendations of Duffie and Skeel [15] and the subsequent finalized stay rule on collateral sale in repo markets, under which collateral can be accessed immediately only if it is held in cash or cash-like assets.

(v) Accelerated payments triggered by defaults. Shortly after Lehman Brothers filed for 
bankruptcy, its counterparties terminated approximately 733,000 of over 900,000 OTC derivatives contracts (Fleming and Sarkar [22]). A contract termination creates a new payment obligation, equal to the market value of the contract, from the out-of-the-money party to the in-the-money party. Lehman's counterparties generally terminated contracts with positive value to the surviving party and chose not to terminate contracts with positive value to Lehman. We expand our model to compare this type of selective termination (known as "cherry-picking") with full termination.

Incorporating accelerated payments through contract termination complicates the analysis, again because one node can benefit from the failure of another, in this case because a default by one node may accelerate payments to other nodes. Arriving at a well-defined set of clearing payments requires making further assumptions on the timing of events; we assume a delay between the failure of a node and any accelerated payments resulting from that failure. This modeling choice is supported by the unfolding of the Lehman bankruptcy in 2008. Claims against Lehman resulting from contract termination became part of the bankruptcy process, leading to delays in payments, as discussed in Fleming and Sarkar [22].

(vi) Alternative contract termination rights. Using the framework of accelerated payments, we compare networks under different contract termination protocols and discuss these comparisons in the context of post-crisis automatic stay rules $2^{2}$ on access to collateral upon a counterparty's failure. We compare three scenarios: selective termination (cherry-picking) by surviving nodes, full termination of all contracts with a failed node, and no termination. We show that selective termination ordinarily results in fewer defaults; but we also show that full termination can reduce systemwide payment shortfalls under a constraint on a measure of firms' derivatives leverage. We make a similar comparison of full-termination and no-termination scenarios. These comparisons are motivated by continuing discussions on the treatment of derivatives in the bankruptcy and resolution of large financial institutions. See Jackson [31], Ghamami [26], the U.S. Department of the Treasury Report [44, and the references therein.

Section 2 reviews the Eisenberg-Noe model, explains the difficulties introduced by collateral, and presents our solution; it also examines the case of costless contract termination and collateral pooling. Section 3 introduces illiquid collateral and presents the joint solution of clearing payments and market prices for collateral assets, along with the implications for defaults and payment shortfalls. Section 4 extends our model to cover accelerated payments from contract

\footnotetext{
${ }^{2}$ These rules generally apply to what are known as qualified financial contracts (QFCs), which include derivatives and repos. See Duffie and Skeel [15], Skeel 38, and Roe and Adams 34 for legal background on stays for QFCs. See Bolton and Oehmke [6] and Duffie and Wang [16] for corporate finance and game theoretic models of automatic stays and contract termination.
} 
termination. Section 5 compares defaults and payment shortfalls under alternative termination scenarios governing collateral access. We defer all proofs to an appendix.

\section{Network Model}

In this section, we first review the model of Eisenberg and Noe [17] and then introduce collateral.

\subsection{Networks without Collateral}

We consider a network with nodes $\mathcal{N}=\{1, \ldots, N\}$ representing banks or other market participants. (We can also think of one node as representing the outside world.) We use the following notation:

$$
\begin{aligned}
\bar{p}_{i j} & =\text { payment due from } i \text { to } j, i, j \in \mathcal{N} ; \\
c_{i} & =\text { cash held by node } i \in \mathcal{N} ; \\
p_{i j} & =\text { actual payment from } i \text { to } j, i, j \in \mathcal{N} .
\end{aligned}
$$

We refer to $c_{i}$ as cash for brevity; more generally, $c_{i}$ represents the near-term cash value of assets (other than the $\bar{p}_{j i}$ ) available to node $i$ to make payments. We assume that payment obligations are netted so that $\bar{p}_{i j}$ and $\bar{p}_{j i}$ cannot both be strictly positive.

We seek to model default triggered by illiquidity rather than insolvency, and this differentiates our formulation from most interbank network models. In our setting, the claims and obligations $\bar{p}_{i j}$ and cash values $c_{i}$ are not intended to provide a complete accounting of a node's balance sheet; each node would typically have other longer-term assets and liabilities. The $\bar{p}_{i j}$ measure payments due. A node defaults if it does not have the cash to make a payment, even if the total value of its assets exceeds the value of its liabilities. Each $c_{i}$ includes the cash a node could raise by borrowing against longer-term assets.

We imagine that, outside the model, some nodes have experienced an exogenous loss of asset value, and we proceed to evaluate payments made, taking the $c_{i}$ as cash levels after the exogenous shock. Given a collection of payments $p_{i j}$, node $i$ 's cash is given by

$$
A_{i}^{o}=c_{i}+\sum_{k \neq i} p_{k i}
$$

and its payments due are given by

$$
L_{i}=\sum_{k \neq i} \bar{p}_{i k} .
$$


Node $i$ defaults if $A_{i}^{o}<L_{i}$, so the default set is

$$
D=\left\{i: \mathcal{N}: A_{i}^{o}<L_{i}\right\}
$$

If node $i$ defaults, its creditors' claims all have equal priority, and any remaining cash held by node $i$ is paid to the creditors in proportion to their claims. These proportions are given by

$$
a_{i j}^{o}=\bar{p}_{i j} / \sum_{k \neq i} \bar{p}_{i k}, \quad i, j \in \mathcal{N}
$$

For each $i$, we have $\sum_{j \neq i} a_{i j}^{o}=1$.

Clearing payments are characterized by the fixed-point equation

$$
\begin{aligned}
p_{i j} & =\bar{p}_{i j} \wedge a_{i j}^{o} A_{i}^{o} \\
& =\bar{p}_{i j} \wedge\left[c_{i}+a_{i j}^{o} \sum_{k \neq i} p_{k i}\right] .
\end{aligned}
$$

This specification ensures that actual payments never exceed obligations $\left(p_{i j} \leq \bar{p}_{i j}\right)$; all creditors have equal priority, in the sense that they receive payments proportional to their claims in the event of default; and the total payments made $\sum_{j \neq i} p_{i j}$ cannot exceed the available cash $A_{i}^{o}(p)$. Eisenberg and Noe [17] showed the existence of a solution to (4) and also gave conditions for uniqueness. Tarski's [41] fixed-point theorem ensures the existence of a largest and a smallest solution of (4).

\subsection{Networks with Collateral: Round 1}

We now introduce collateral, which we also refer to as initial margin (IM). We let

$$
m_{i j}=\text { margin posted by node } i \text { for obligations to node } j \text {. }
$$

Suppose, for example, that nodes $i$ and $j$ are two banks that have entered into a swap contract. Under rules adopted in the U.S. in 2015 and 2016, each bank is required to post IM as collateral against potential future payments to the other bank.$^{3}$ If node $j$ is a central counterparty (CCP), then $i$ posts IM to $j$, but $j$ does post IM to $i$. Because IM is intended to cover potential future losses, a node will often face a margin requirement on a contract even if no payment is due.

We will refer to $m_{i j}$ as margin posted or committed by node $i$ to node $j$. We assume that the margin $m_{i j}$ remains an asset of node $i$ until node $i$ fails to make a payment to node $j \bigsqcup^{4}$

\footnotetext{
${ }^{3}$ U.S. banking regulators and the CFTC finalized their uncleared swap margin rules in 2015 and 2016 . U.S. margin rules for uncleared swap transactions follow closely the guidelines established by the BCBS-IOSCO [4.

${ }^{4}$ This assumption is consistent with the treatment of IM in practice. The required IM is commonly held by a third-party custodian.
} 
The quantity $m_{i j}$ differs from other assets held by node $i$ in that node $j$ 's claim to the $m_{i j}$ has priority over the claims of any other creditors. We take $m_{i i}=0$ for all $i$.

We assume the following sequence of events at default. Node $i$ goes into default when it has insufficient cash (including payments received from other nodes) to meet its obligations. Rather than make partial payments that would not stave off default, node $i$ briefly suspends making any payments. At this point, node $j$ seizes enough of the collateral $m_{i j}$ to cover any payment due $\bar{p}_{i j}$ from $i$ to $j$. The amount of collateral seized by node $j$ from node $i$ is given by

$$
\Delta_{i j}= \begin{cases}m_{i j} \wedge \bar{p}_{i j}, & i \in D \\ 0, & i \notin D\end{cases}
$$

In particular, if node $i$ defaults, node $j$ 's claim to the collateral $m_{i j}$ is determined solely by the payment obligation $\bar{p}_{i j}$ and is unaffected by any other claims on node $i$ 's assets. This is the defining feature of collateral.

If the margin seized by node $j$ from node $i$ is insufficient to cover the obligation $\bar{p}_{i j}$, node $j$ retains a residual claim of $\bar{p}_{i j}-m_{i j}$, which has equal priority with any residual claims against $i$ by other nodes. This claim will produce a partial payment from $i$ to $j$ if node $i$ has any remaining assets. To reflect a pro rata allocation of node $i$ 's cash to these equal-priority claims, we replace $(3)$ with the proportion: $5^{5}$

$$
a_{i j}^{(1)}=\left[\bar{p}_{i j}-m_{i j}\right]^{+} / \sum_{k \neq i}\left[\bar{p}_{i k}-m_{i k}\right]^{+}, \quad i, j \in \mathcal{N} .
$$

If the denominator is zero, no node has a residual claim on $i$, and we may set $a_{i j}^{(1)} \equiv 0$; if node $i$ does not default, set $a_{i j}^{(1)}=a_{i j}^{o}$, as in 3 .

Let $p_{i j}^{(1)}$ denote the total payment made by node $i$ to node $j$, consisting of any collateral seizure $\Delta_{i j}$ and any partial payments based on the proportions 66 . Given payments $p_{k i}^{(1)}$ made to node $i$, the cash available to node $i$ is given by

$$
A_{i}^{(1)}=c_{i}+\sum_{k} p_{k i}^{(1)}
$$

this expression has the same form as (1), but it includes node $i$ 's access to collateral $m_{k i}$ posted by other nodes and seized according to (5). The set of defaulting nodes is given by

$$
D=\left\{i \in \mathcal{N}: A_{i}^{(1)}<L_{i}\right\}
$$

\footnotetext{
${ }^{5}$ The assumption that collateral is taken first and the bankrupt firm's assets are distributed in proportion to the residual claims is consistent with the legal discussion in Ayer, Bernstein, and Friedland [2].
} 
with the sets $L_{i}$ as in 2 . The requirements for first-round clearing payments $p_{i j}^{(1)}$ now take the form

$$
p_{i j}^{(1)}= \begin{cases}\bar{p}_{i j} \wedge\left[m_{i j}+a_{i j}^{(1)} A_{i}^{(1)}\right], & i \in D ; \\ \bar{p}_{i j}, & i \notin D .\end{cases}
$$

These conditions extend (4) using (6)-(8) and including the collateral $m_{i j}$. If, for some $j$, $m_{i j}+a_{i j}^{(1)} A_{i}^{(1)}<\bar{p}_{i j}$, then $a_{i j}^{(1)} A_{i}^{(1)}<\bar{p}_{i j}$, and node $i$ is in default. We may therefore write $(9)$ as

$$
p_{i j}^{(1)}=\bar{p}_{i j} \wedge\left[m_{i j}+a_{i j}^{(1)} A_{i}^{(1)}\right] .
$$

This expression is similar to the Eisenberg-Noe equation (4), but the similarity hides an important difference: we cannot recover the default set from payments in (10) because a node in default may meet its payment obligations through access to collateral. In (4), $p_{i j}=\bar{p}_{i j}$ implies that node $i$ had not defaulted, but we cannot make a similar inference from $p_{i j}^{(1)}=\bar{p}_{i j}$ in 10 .

We will confirm the existence of clearing payments $p^{(1)}=\left\{p_{i j}^{(1)}, i, j \in \mathcal{N}\right\}$ satisfying $(6)-(10)$ shortly, but we first elaborate on the potential complications introduced by collateral.

\section{$2.3 \quad$ Freed Collateral: Round 2}

Following a default by node $i$, it may happen that the collateral committed to node $j$ exceeds the payment obligation to $j$, in which case the excess $m_{i j}-\Delta_{i j}$ would become available to node $i$ to meet other obligations. If another node $k$ also defaults, then margin $m_{i k}$ posted by $i$ to $k$ may also become available to $i$. This return of collateral is potentially problematic for the existence of clearing payments, as the following example illustrates.

Example 2.1. Consider the network illustrated in Figure 1, with nodes A, B, and C. The number inside each circle indicates the node's cash; all cash levels are initially zero. The arrows indicate the directions of payment obligations, and the labels on the arrows indicate the amounts due. The labels in square brackets show posted margin. For example, the label "[5-A]" above node $\mathrm{C}$ indicates that node $\mathrm{A}$ has posted collateral worth 5 to node $\mathrm{C}$.

As no node has cash, all nodes default. The default of node $\mathrm{C}$ returns collateral worth 5 to A, and similarly B and $\mathrm{C}$ each recover collateral worth 5 from the defaults A and B. The result is the configuration in the middle of the figure. With the freed collateral, all nodes can meet their payment obligations, resulting in the final configuration.

But if all nodes have met their payment obligations, have they defaulted? It is not possible in this example to simultaneously determine a consistent set of payments and default designations. In the rightmost configuration, no node appears to be in default, but we cannot reach that configuration without freeing collateral, which requires defaults. 

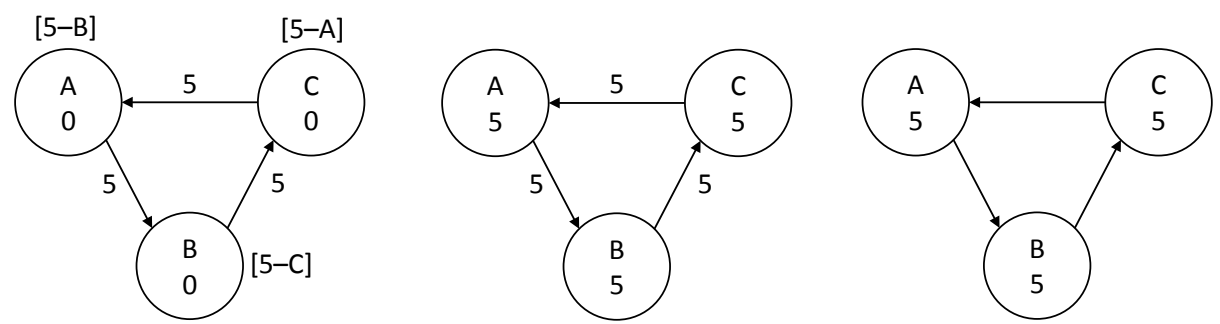

Figure 1: A three-node network. All nodes start with zero cash. The labels in brackets show collateral — the amount posted and the posting party.

The problem illustrated by this example is that the default of one node can lead to the return of collateral to other nodes. Those nodes may then be able to make greater payments to the defaulted node, potentially lifting that node out of default, and precluding the existence of an internally consistent set of payments ${ }^{6}$

We avoid this difficulty through the definition of the default set in (8), based on $p^{(1)}$. In this formulation, freed collateral does not become available until after payments have been made and nodes have been declared in default. We have thus separated the payments process into two rounds: a first round in which payments are made and collateral is seized as needed; and a second round in which excess collateral and collateral posted to defaulted nodes becomes available to the posting party to make other payments.

Obstacles to defining clearing payments arise in other extensions of Eisenberg and Noe [17] as well. Elsinger [18] redefines clearing vectors to cover cross-holdings of debt and equity among banks; David and Lehar [12] consider clearing payments when debt is subject to renegotiation; Kusnetsov and Veraart [32] propose a detailed algorithm to handle debt with different maturities; Jackson and Pernoud [30] note that clearing payments may not be well-defined when banks buy credit protection on other banks, and Banerjee and Feinstein [3] similarly preclude banks from speculating on other banks. None of these extensions fits our setting. Bichuch and Feinstein [5] consider networks with collateral, but in their setting banks post collateral outside the network to raise cash; in particular, they do not allow one bank to seize collateral posted by another bank, which is a key feature of collateral in our setting.

In Figure 1, no collateral is seized because collateral is sitting at the wrong nodes to secure payments due. For example, C owes A, but A has posted collateral to C. As a result, all collateral is freed when the nodes default. Figure 2 shows the opposite configuration, with the

\footnotetext{
${ }^{6}$ Chang [9] develops a model in which borrowers may fail to recover collateral when a lender defaults because the lender has in turn posted the collateral to another node. In our analysis, we assume nodes fully recover any collateral to which they are entitled.
} 

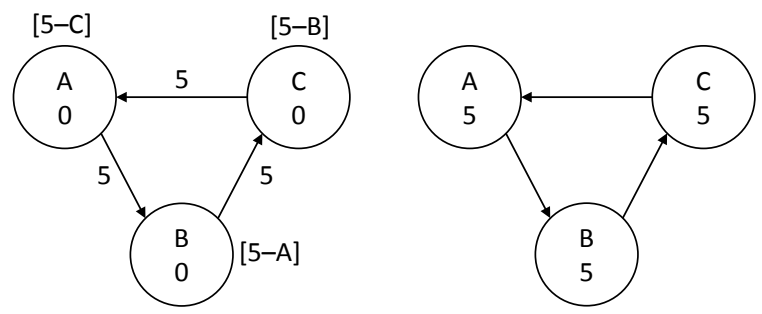

Figure 2: The direction of collateral is reversed relative to Figure 1

direction of collateral postings reversed. All nodes default, all nodes seize collateral worth 5 to cover the amount owed, and there are no remaining payment obligations. As in Figure 1, there is no way to simultaneously define clearing payments and the default set: if all nodes default, then all payments are covered, so no node defaults; if no node defaults, then no collateral is seized, no payments are covered, so all nodes default.

The return or freeing of collateral in Figure 1 implicitly assumes that contracts with defaulted nodes are automatically terminated. Recall that margin $m_{i j}$ is intended to cover future potential losses on a contract (e.g., a swap), so it is possible to have margin posted when no payment is due - that is, $m_{i k}>0$ and $\bar{p}_{i k}=\bar{p}_{k i}=0$. If node $k$ defaults on its payments to other nodes, the implications for node $i$ are unclear. We assume (for now) that any contracts between $i$ and $k$ are canceled and that posted collateral is returned: $m_{i k}$ becomes available to node $i$ and $m_{k i}$ becomes available to node $k$. We examine alternative assumptions on contract termination and collateral access later.

Suppose the first round results in payments $p_{i j}^{(1)}, i, j \in \mathcal{N}$. In other words, the $p_{i j}^{(1)}$ satisfy (6)-(9). If the payment due from $i$ to $j, \bar{p}_{i j}$ exceeds the payment $p_{i j}^{(1)}$, then node $i$ enters the second round with a remaining payment obligation to node $j$ of

$$
\bar{p}_{i j}^{(2)}=\bar{p}_{i j}-p_{i j}^{(1)} .
$$

In Round 2, we deal with the allocation of remaining resources to meet remaining payment obligations. These remaining resources result from freed collateral.

In the Eisenberg-Noe setting, (4) ensures that a node in default pays out all its cash. But the use of collateral in Round 1 makes two types of resources potentially available at a defaulted node in Round 2: a node may recover excess collateral committed to another node; and it may recover freed collateral committed to a defaulting node, as a result of contract termination. Figure 3 illustrates these mechanisms.7

\footnotetext{
${ }^{7}$ It is also possible for node $i$ to default in Round 1 yet end the round holding cash, even before the return of
} 

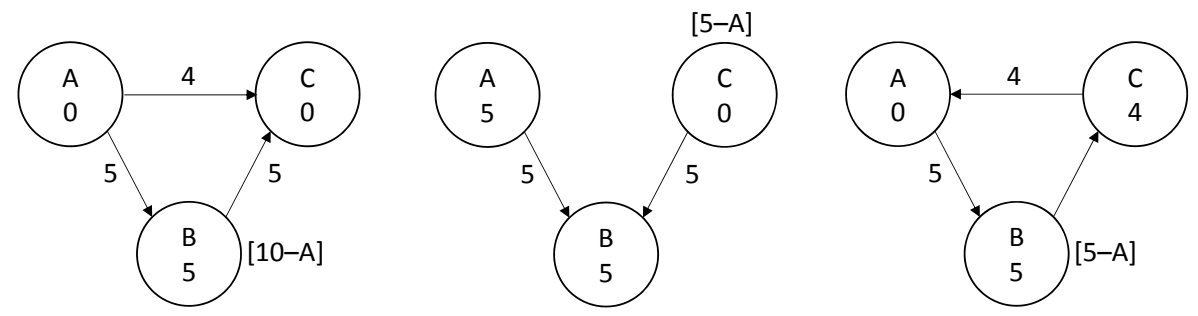

Figure 3: Three initial configurations, each leaving A with cash. In the first network, excess collateral is returned to A following A's default; in the second network, contract termination returns collateral to $\mathrm{A}$; in the third network, $\mathrm{A}$ is left with cash despite defaulting on its payment to B.

Example 2.2. Figure 3 shows three starting configurations. In the first network, A defaults, B seizes collateral to cover missed payments from A, and excess collateral is returned to A, allowing A to pay C. In the second network, C defaults on its obligation to B; C's default cancels its contract with $\mathrm{A}$, freeing the collateral posted by $\mathrm{A}$ to $\mathrm{C}$, leaving $\mathrm{A}$ with 5 in cash. In the last network, A defaults, B seizes its collateral, and A is left with 4 units of cash, despite having defaulted. See Section 2.4 for a discussion of full repayment following default.

The total collateral "returned" to node $i$ after Round 1 is given by

$$
r_{i}= \begin{cases}\sum_{j \neq i}\left(m_{i j}-\Delta_{i j}\right), & i \in D ; \\ \sum_{j \in D} m_{i j}, & i \notin D .\end{cases}
$$

with $D$ fixed by (8) in Round 1. If this freed collateral is insufficient to meet all of node $i$ 's remaining claims, payments are made in the proportions

$$
a_{i j}^{(2)}=\bar{p}_{i j}^{(2)} / \sum_{k \neq i} \bar{p}_{i k}^{(2)}
$$

taking $a_{i j}^{(2)}=0$ if the denominator is zero. Thus, we seek Round 2 payments satisfying

$$
p_{i j}^{(2)}=\bar{p}_{i j}^{(2)} \wedge a_{i j}^{(2)}\left(r_{i}+\sum_{k \neq i} p_{k i}^{(2)}\right), \quad i, j \in \mathcal{N} .
$$

For later use, we record a relationship between the allocation fractions in the two rounds.

Lemma 2.1. If $a_{i j}^{(2)} \neq 0$ then $a_{i j}^{(2)}=a_{i j}^{(1)}$.

In the following, we use $p_{i}^{(\ell)}=\sum_{j} p_{i j}^{(\ell)}, \ell=1,2$, to denote the total amount paid by node $i$ in each round.

collateral. However, in this case, we would have $\bar{p}_{i j}^{(2)}=0$ for all $j$ and node $i$ has no remaining payments. This case is also illustrated in the figure. 
Proposition 2.1. For any collateral levels $\left\{m_{i j}, i, j \in \mathcal{N}\right\}$, there exist clearing payments $\left(p^{(1)}, p^{(2)}\right)$ satisfying (6)-(9) and (11)-(14). Moreover,

$$
p_{i}^{(1)}+p_{i}^{(2)}=\bar{p}_{i} \wedge\left[A_{i}\left(p^{(1)}+p^{(2)}\right)+\sum_{k \neq i} m_{i k}\right],
$$

where

$$
A_{i}\left(p^{(1)}+p^{(2)}\right)=c_{i}+\sum_{k \neq i}\left(p_{k i}^{(1)}+p_{k i}^{(2)}\right)
$$

As our examples have illustrated, the existence of clearing payments depends on separating the timing of payments due from the freeing of collateral: the default set $D$ and returned collateral $r_{i}$ are determined by the first-round payments $p^{(1)}$. Without this separation, the network will often fail to admit a consistent set of clearing payments. Equation (15) has a simple interpretation: the total payments made by a node over two rounds equals the lesser of the node's total obligations and the node's total cash, including collateral. However, (15) does not extend to node-specific payments $p_{i j}^{(1)}+p_{i j}^{(2)}$ because collateral is initially committed to specific counterparties.

In subsequent sections, we will compare outcomes of networks under different policies regarding collateral and contracts. We will make these comparisons based on the sets of defaulting nodes and the system-wide payment shortfall, which builds on the total payments in (15).

Definition 2.1. A network's payment shortfall is the difference between payments due and payments made, given by

$$
L=\sum_{i}\left(\bar{p}_{i}-p_{i}^{(1)}-p_{i}^{(2)}\right)=\sum_{i \in D}\left(\bar{p}_{i}-p_{i}^{(1)}-p_{i}^{(2)}\right) .
$$

For each node $i$ that defaults, $\bar{p}_{i}-p_{i}^{(1)}-p_{i}^{(2)}$ is the difference between $i$ 's total payment obligation and its total payments; if $i$ does not default, then $\bar{p}_{i}=p_{i}^{(1)}, p_{i}^{(2)}=0$, and its shortfall is zero.

Table 1 shows the default sets and payment shortfalls for the examples in several figures. In several cases, we have designed the examples to have $L=0$ to highlight the effect of two rounds of payments. In the first example of Figure 3 , for instance, increasing node A's payment obligations from 9 to $10+x$ would result in $L=x$, for any $x \geq 0$, without changing $D$.

To see that our two rounds cannot be combined in general, consider an ordinary EisenbergNoe network without collateral. Suppose that for some node $i$ there exists a cash level $\underline{c}$ such that $i$ defaults if $c_{i}<\underline{c}$, and $i$ does not default if $c_{i}>\underline{c}$. (We can see from (7) and (8) that such 


\begin{tabular}{l|l|lll|l|l}
\multicolumn{1}{c}{ Fig. 2} & \multicolumn{1}{c}{ Fig. 3} & \multicolumn{1}{c}{ Fig. 6} \\
\hline$D=\{A, B, C\}$ & $D=\{A, B, C\}$ & $D=\{A\}$ & $D=\{C\}$ & $D=\{A\}$ & $D=\{A\}$ & $D=\{A\}$ \\
$L=0$ & $L=0$ & $L=0$ & $L=5$ & $L=0$ & $L=9$ & $L=5$ \\
\hline
\end{tabular}

Table 1: Default sets $D$ and payment shortfalls $L$ for examples in the figures. Figure 3 has three examples.

thresholds will commonly exist.) Now introduce collateral and consider any proposed protocol with the following two intended features: (i) collateral posted by a node is used towards the node's payment obligations if and only if that node defaults, and (ii) a node is deemed to default if and only if it fails to meet its payment obligations. Such a protocol is not in general consistent:

Proposition 2.2. Suppose $\sum_{j} m_{i j}>\underline{c}-c_{i}>0$, and suppose $m_{k \ell}=0$, for all $k \neq i$. If node $i$ defaults, then it meets all its payment obligations; if node $i$ does not default then it does not meet all its payment obligations. In other words, no choice of default set $D$ is consistent with the protocol.

The proof of this claim is simple: If node $i$ is deemed to default, then the resources $c_{i}+$ $\sum_{j} m_{i j}>\underline{c}$ suffice to meet the node's obligations, along with any cash received from other nodes. If node $i$ does not default, then the cash level $c_{i}<\underline{c}$ does not suffice for $i$ to make its payments. In either case, we have a contradiction. Similar contradictions result from many other configurations.

\subsection{Default with Full Repayment}

We have seen (as in Table 1) that it is possible for a node to default in the first round yet fully meet its payment obligations by the end of the second round, eliminating its first-round shortfall. In other words, a node may fail through illiquidity - a shortage of cash to meet payments due - even if it is solvent, because some of its assets have been pledged as collateral. Indeed, the major failures and near-failures of 2008 are generally understood as (at least initially) crises of liquidity rather than solvency. Post-crisis regulation responded by introducing a liquidity coverage ratio for banks as a complement to traditional capital requirements.

One may ask whether a default is costly if creditors are ultimately repaid. This question goes to our definition of the default set, so we highlight two important practical considerations that inform our modeling choice. First, a delay in payments can be costly because of its ripple effect on downstream parties that rely on receiving those payments to meet their own obligations. Delays can also lead to credit downgrades which then affect a node's ability to borrow. Second, 
a delay that results in bankruptcy destroys franchise value; it locks a failed firm out of markets that rely on a solid reputation for meeting payment obligations on time, and such a reputation is not restored simply through eventual repayment. Nor are other costs of bankruptcy recovered. The specifics of these mechanisms are beyond the scope of our model, but these considerations explain why we treat first-round defaults as costly, even when second-round obligations are fully met.

\subsection{Networks with a Free Termination Option}

As a benchmark, we consider a variant of our model in which nodes have the option to terminate contracts in order to redeploy collateral they have posted. This termination option is exercised whenever a node would otherwise default. We investigate other contract termination scenarios more extensively in Sections 4 and 5 .

To motivate this variant, consider the three networks in Figure 3 . Suppose that node A has the option to terminate any contract by using collateral posted to pay its counterparty, recovering any remaining collateral. In the first case, this would mean paying 5 to $\mathrm{B}$ and recovering 5 ; in the second case, paying zero to $\mathrm{C}$ and recovering 5 ; in the third case, paying 5 to $\mathrm{B}$ and recovering zero. In each case, A would avoid default.

We show in Appendix A.3 that this model can be reduced to a standard Eisenberg-Noe model with lower payment obligations and adjusted cash balances in the following sense:

Lemma 2.2. Payments $p_{i j}$ clear the network with collateral and free contract termination if and only if payments $p_{i j}-\left(m_{i j} \wedge \bar{p}_{i j}\right)$ clear the reduced Eisenberg-Noe network. The two networks have the same default sets and payment shortfalls.

The reduced network is defined precisely in Appendix A.3. This result shows that collateral plays no essential role in a network in which each node can recover collateral by terminating contracts. With the option to terminate, posting collateral reduces to paying down certain obligations. This result allows us to compare the collateralized networks of Sections $2.2,2.3$ with otherwise identical networks that allow free contract termination: 8

Proposition 2.3. Free contract termination reduces defaults but does not affect total payment shortfalls.

\footnotetext{
${ }^{8}$ Here and in several subsequent results we are comparing two networks defined by fixed-point equations. To account for the possibility of multiple fixed points, the comparison should be understood to hold for the largest fixed points of the two networks and for the smallest fixed points. The existence of largest and smallest fixed points follows from applications of Tarski's fixed-point theorem. These issues are discussed in greater detail in the proofs of the relevant results.
} 
This comparison confirms the natural intuition that collateral trapped in the "wrong" places will increase defaults (because it is not immediately available to meet payment obligations) but not affect eventual payments (because excess collateral is eventually returned and deployed to make payments).

The free-termination model is useful for illustration, but it overlooks two key points. First, parties to swaps and similar contracts do not ordinarily have the right to terminate contracts unilaterally 9 but must pay for that option by, for example, buying a swaption. The issue of termination near default can be particularly contentious, a point we return to in Section 5 . Furthermore, in the second example of Figure 3, suppose we change A's obligation to B to 8 and change C's obligation to B to 3 . Node A would like to terminate its contract with $\mathrm{C}$ to recover its collateral. But $\mathrm{C}$ has no reason to agree and would like to extract a payment from A. To avoid default, both nodes would like to keep at least 3 units of the 5 units of collateral.

The second key point missed by this model variant is the distinction between payments due and contract values. Suppose node A has taken out a loan of 50 from node C and posted 5 in collateral. In the middle example of Figure 3, no interest is due on the loan. But if A were to terminate the contract to recover its collateral, it would need to repay the principal, dramatically increasing its immediate payment obligations. The same can happen with a cross-currency swap, which entails an exchange of principal at maturity. We consider these consequences of contract termination in detail in Section 4. For these reasons, we work with the framework of Sections 2.2 2.3 for the rest of the paper: collateral becomes accessible only following a default.

\subsection{Collateral Pooling}

In the examples of Figures 2 and 3, some defaults occur because collateral is tied up in the "wrong" places. More precisely, some defaults could be avoided if nodes were able to hold on to their collateral as cash, rather than commit it to specific counterparties. This points to a tradeoff similar to the tradeoff between capital requirements and collateral requirements: collateral provides a buffer against specific losses, whereas capital absorbs any type of loss. Similar considerations apply in debates over "ring-fencing" capital to absorb losses in specific jurisdictions as opposed to holding capital at the parent level.

These considerations might suggest that defaults and losses can be reduced by having all nodes hold additional cash rather than post collateral. But that conclusion is incorrect because changing the distribution of collateral changes the distribution of payments.

\footnotetext{
${ }^{9}$ Most OTC derivatives contracts are governed by an ISDA Master Agreement, which specifies the very limited settings - primarily events of default by the counterparty - in which one party may terminate a contract. See the discussion in Appendix B
} 


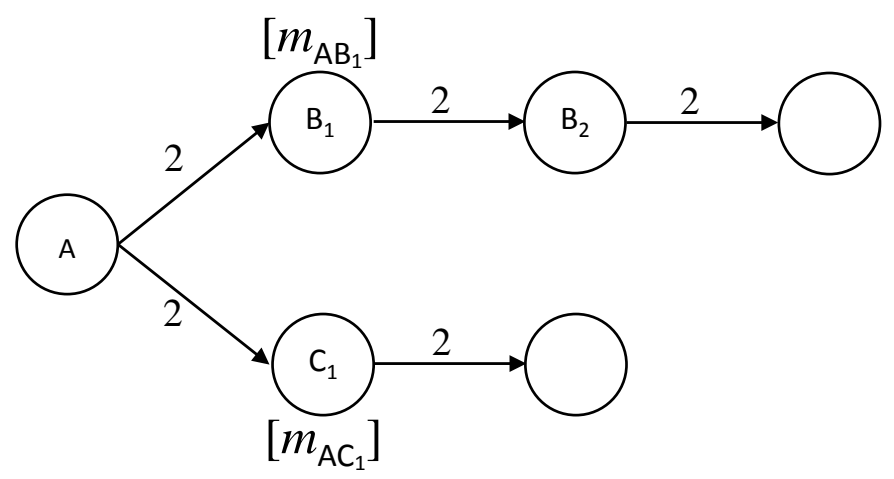

Figure 4: Pooling collateral may increase or decrease payment shortfalls and the number of defaults

Consider the example of Figure 4. The labels on the edges indicate payment obligations. None of the nodes holds cash, but node $A$ may have posted collateral to $B_{1}$ or $C_{1}$. We compare defaults and shortfalls in the following scenarios:

$$
\begin{array}{ll}
c_{A}=0, m_{A B_{1}}=0, m_{A C_{1}}=2: & D=\left\{A, B_{1}, B_{2}\right\}, L=6 ; \\
c_{A}=2, m_{A B_{1}}=0, m_{A C_{1}}=0: & D=\left\{A, B_{1}, B_{2}, C_{1}\right\}, L=5 ; \\
c_{A}=0, m_{A B_{1}}=2, m_{A C_{1}}=0: & D=\left\{A, C_{1}\right\}, L=4 .
\end{array}
$$

In the first scenario, collateral is committed to node $C_{1}$. Holding the collateral as cash instead, as indicated in the second scenario, results in a payment of 1 to each of $B_{1}$ and $C_{1}$. This increases the number of defaults but it reduces systemwide payment shortfalls, which argues in favor of pooling. But the third configuration, with collateral committed to node $B_{1}$, yields the fewest defaults and the smallest shortfall.

As this example illustrates, pooling collateral is not unambiguously better or worse than committing it to specific counterparties. The comparison depends on the network and cannot be resolved by considering a node in isolation.

The next result shows that pooling is preferable in two settings. To be precise, we need some terminology. Let us say that one network is obtained from another by pooling collateral if it results from one or more transformations of the form $m_{i j}^{\prime}=m_{i j}-\delta, c_{i}^{\prime}=c_{i}+\delta, \delta \leq m_{i j}$. By the excess collateral posted by node $i$ to node $j$ we mean $\left[m_{i j}-\bar{p}_{i j}\right]^{+}$. We say that the network has proportional collateral if $m_{i j}=k_{i} \bar{p}_{i j}$, for some $k_{i} \in[0,1]$, for all $i$ and $j$. (The case $k_{i}>1$ would be a special case of excess collateral.)

Proposition 2.4. (i) Pooling excess collateral reduces defaults and does not affect payment shortfalls. (ii) Under proportional collateral, pooling that preserves proportionality reduces de- 
faults and does not affect payment shortfalls.

This proposition and the example of Figure 4 together suggest that pooling is unambiguously better (in reducing defaults and shortfalls) only under special conditions. A proportional collateral rule (like a fixed loan-to-value ratio) is found in some circumstances, but it is not applicable with derivative contracts that carry different levels of risk or when payment obligations are not known precisely at the time collateral is posted. Excess collateral is applicable to derivative contracts as collateral in the form of initial margin can exceed current contractual payment obligations. Since initial margin in part captures extreme potential future exposures, it can exceed current payments, making the excess collateral condition particularly relevant to OTC derivatives markets.

\section{Illiquid Collateral and Fire Sales}

In Sections 2.2 and 2.3, we implicitly treated collateral as cash: if node $j$ seizes collateral $\Delta_{i j}$ from node $i$, node $i$ 's payment obligation is reduced by exactly $\Delta_{i j}$. In the non-cleared derivatives market, a wide range of less liquid securities, including corporate bonds, foreigndenominated bonds, and equities are accepted as collateral, and these types of securities are also used as collateral in repurchase agreements.

In this section, we extend our earlier analysis to incorporate the use of less liquid collateral. When a creditor seizes collateral, it must sell the collateral to recover cash. Selling less liquid collateral drives down its price, spreading losses to other holders of similar assets. Moreover, if excess collateral is available, the creditor has no incentive to sell at the best possible price, making the risk of a fire sale particularly acute.

Indeed, collateral liquidity is at the heart of debates over contract termination rights and bankruptcy stays that motivate our investigation. As explained, for example, in Roe and Adams [34], p.366, the purpose of bankruptcy stays is to avoid the value destruction and fire sales that would occur if creditors were allowed to cease and sell a failing firm's illiquid collateral. Regulators and industry participants continue to debate the extent to which less liquid collateral should be allowed for derivatives and repo, and whether the nature of the collateral necessitates different restrictions on contract termination and rules on stays.

\subsection{Round 1 Revisited}

As a first step, we reformulate the analysis of Sections 2.22 .3 to incorporate illiquidity. We now take $m_{i j}$ to be the shares of collateral committed by node $i$ to node $j$ for an asset with price $\pi$, 
making $m_{i j} \pi$ the value of the collateral. The case considered in Sections 2.22 .3 corresponds to a constant value $\pi=1$. In this section, the price starts at 1 but falls as collateral is liquidated.

We posit that $\pi$ is a strictly decreasing function $G(1, \Delta)$ of the total shares sold, $\Delta$, the first argument of $G$ indicating the initial price of 1 . To be concrete, we set

$$
\pi=G(1, \Delta) \equiv e^{-\alpha \Delta}
$$

for some $\alpha \geq 0$. A larger $\alpha$ corresponds to a less liquid asset. This choice of price-impact function is also used in Cifuentes et al. [10]; Amini et al. [1] discuss conditions on $\alpha$.

We make the simplifying assumption that all collateral is held in a single illiquid asset. Assigning different prices and price-impact functions to different collateral assets would complicate notation without significantly changing our analysis. Assuming a single illiquid asset for all collateral overstates the effect of fire sales, but the overstatement can be offset through a smaller value of $\alpha$. The choice of $\alpha$ should reflect the average price impact across different types of collateral and the imperfect correlation in price impact across different securities.

As in (7) and (8), we have

$$
A_{i}^{(1)}=c_{i}+\sum_{k} p_{k i}^{(1)}, \quad D=\left\{i: A_{i}^{(1)}<\sum_{k} \bar{p}_{i k}\right\}
$$

but the payments in $A_{i}^{(1)}$ received by node $i$ will now reflect the market price of the collateral asset. If node $i$ defaults and node $j$ seizes collateral $m_{i j}$ with price $\pi$, node $j$ now holds a residual claim of $\left(\bar{p}_{i j}-\pi m_{i j}\right)^{+}$against node $i$, reflecting the market value $\pi m_{i j}$ of the collateral available. Node $i$ 's assets are allocated to other nodes in proportion to the values of these claims, so we replace (6) with

$$
a_{i j}^{(1)}=\left(\bar{p}_{i j}-\pi m_{i j}\right)^{+} / \sum_{k \neq i}\left(\bar{p}_{i k}-\pi m_{i k}\right)^{+} .
$$

Example 3.1. Figure 5 illustrates the difference between allocation proportions (6) and (19). Node A has 6 in cash and 20 in payment obligations, so it defaults. Node B seizes the 5 shares of the collateral asset posted by A. The 6 in cash held by A is divided between B and $\mathrm{C}$. Under (6), B would be allocated a proportion $(10-5) / 15=1 / 3$, and $\mathrm{C}$ a proportion $2 / 3$. Under (19), node $\mathrm{B}$ claims a proportion $(10-5 \pi) /(20-5 \pi)$, which is a decreasing function of the price $\pi$ at which $B$ sells collateral it seized from A; the allocation proportions depend on the market price of the collateral asset.

With asset price $\pi>0$, the shares of collateral seized and sold by node $j$ upon the default of node $i$ are given by

$$
\Delta_{i j}= \begin{cases}m_{i j} \wedge \frac{\bar{p}_{i j}}{\pi} ; & i \in D \\ 0, & i \notin D\end{cases}
$$




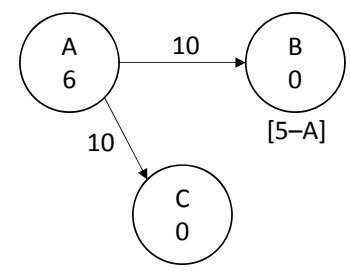

Figure 5: After A defaults, B seizes its collateral, and A's 6 units of cash are divided between $\mathrm{B}$ and $\mathrm{C}$.

which reduces to (5) with $\pi=1$. Dividing by $\pi$ in (20) converts the dollar obligation $\bar{p}_{i j}$ into the number of shares required to cover the payment at the current market price. For $\pi=0$, interpret (20) as $\Delta_{i j}=m_{i j}$ if $\bar{p}_{i j}>0$ and $\Delta_{i j}=0$ otherwise. The total shares of collateral liquidated are given by

$$
\Delta=\sum_{i} \sum_{j} \Delta_{i j}
$$

and the sale of these shares will drive down the price through (17). We modify (9) by seeking clearing payments $p^{(1)}$ and an asset price $\pi^{(1)}$ satisfying

$$
p_{i j}^{(1)}= \begin{cases}\bar{p}_{i j} \wedge\left[\pi^{(1)} m_{i j}+a_{i j}^{(1)} A_{i}^{(1)}\right], & i \in D ; \\ \bar{p}_{i j}, & i \notin D,\end{cases}
$$

together with $(17)-(21)$.

\subsection{Round 2 Revisited}

Suppose that Round 1 clears with payments $p^{(1)}$ and asset price $\pi^{(1)}$. Define $r_{i}$ as in $(12)$, and interpret it as the number of shares of collateral freed or returned to node $i$. As in (11), node $i$ 's remaining obligation to node $j$ is given by $\bar{p}_{i j}^{(2)}=\bar{p}_{i j}-p_{i j}^{(1)}$.

To meet its remaining obligations $\bar{p}_{i j}^{(2)}$, node $i$ will liquidate some or all of its freed collateral, which will further drive down the price of the shares. Given second-round payments $p_{i j}^{(2)}$ and a second-round share price $\pi^{(2)}>0$, the number of shares liquidated by node $i$ is given by

$$
\Gamma_{i}=r_{i} \wedge \frac{1}{\pi^{(2)}}\left(\sum_{j \neq i} \bar{p}_{i j}^{(2)}-\sum_{j \neq i} p_{j i}^{(2)}\right) .
$$

The expression in parentheses is the difference between node $i$ 's remaining payment obligations and the second-round payments it receives; dividing by $\pi^{(2)}$ yields the number of shares of collateral required to make up this shortfall; but node $i$ cannot liquidate more than the $r_{i}$ shares it recovers. The total amount liquidated by all nodes is $\Gamma=\sum_{i} \Gamma_{i}$, driving the price to

$$
\pi^{(2)}=G\left(\pi^{(1)}, \Gamma\right)=\pi^{(1)} e^{-\alpha \Gamma} .
$$


To clear Round 2, we need payments $p^{(2)}$ and a price $\pi^{(2)}$ satisfying

$$
p_{i j}^{(2)}=\bar{p}_{i j}^{(2)} \wedge a_{i j}^{(2)}\left(\sum_{k \neq i} p_{k i}^{(2)}+\pi^{(2)} r_{i}\right),
$$

together with $23-24)$, and $a_{i j}^{(2)}$ as in 13 . The following result ensures the existence of clearing payments and compares networks with liquid and illiquid collateral.

Proposition 3.1. There exist clearing payments and prices $\left(p^{(1)}, \pi^{(1)}\right)$ and $\left(p^{(2)}, \pi^{(2)}\right)$ for Rounds 1 and 2 with illiquid collateral.

Two features in particular distinguish this result from other network models with fire sales, such as Cifuentes et al. [10, Braouezec and Wagalath [7], and Cont and Schaanning [11]: one is the need to split the payments into two rounds because of the collateral, and the second is the fact that the proportions (19) depend on the collateral price $\pi$. Both features lead to more involved arguments for the existence of clearing payments and prices. The source of the fire sale is also different. In prior work, the fire sale is driven by banks selling their own assets to meet capital requirements; in our setting, the fire sale is driven by creditors selling collateral to recover payment shortfalls.

Our next result compares networks with liquid and illiquid collateral. In stating the result, we need to account for the possibility that each network admits multiple sets of clearing payments. We will show that each network has a largest and smallest set of first-round and total payments $\left(p^{(1)}, p^{(1)}+p^{(2)}\right)$. The following comparison should be understood to hold for the largest and smallest solutions of networks with liquid and illiquid collateral:

Proposition 3.2. Collateral illiquidity increases defaults and the total payment shortfall.

\subsection{Collateral Fire Sale and Contagion}

Our model formulation in Sections 2.2 and 3.1 assumed that, upon a default in Round 1, collateral is seized first and partial payments are made second. For comparison, in this section we consider an alternative formulation in which access to collateral is delayed and partial payments are made first. Within Round 1, we reverse the order of collateral seizure and partial payments; Round 2 proceeds as before. This reversal may be interpreted as the result of an automatic stay, in which a defaulting node's counterparties are prevented from immediately seizing and liquidating collateral. (We discuss stays in greater detail in Section 5.) We give an example here and leave the details for Appendix A.7. We show there that in the absence of fire sales the total payments made from one node to another remain unchanged under this 


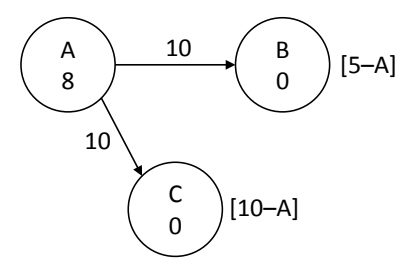

Figure 6: Nodes B and C hold collateral posted by A. Under the payments-first protocol, A pays 8 to B before collateral is seized. Under the collateral-first protocol, A pays 5 to B after collateral is seized.

protocol, even though the mix of collateral and cash payments may change. With illiquid collateral, delaying collateral liquidation reduces systemwide losses and defaults.

Figure 6 illustrates the result. Node A defaults because its cash level 8 falls below its payment obligations of 20 . Under the collateral-first protocol, nodes B and C seize and liquidate the collateral posted by A, which is $m_{A B}=5$ and $m_{A C}=10$. Applying 10 with $a_{A B}^{(1)}=1$ and $a_{A C}^{(1)}=0$, node A makes a cash payment of 5 to node $\mathrm{B}$ in addition to the collateral transfer of 5. The total first-round payments are $p_{A B}^{(1)}=p_{A C}^{(1)}=10$, and there are no second-round payments. Under the payments-first protocol, node A first makes a cash payment of 8 to node $\mathrm{B}$, again because $a_{A B}^{(1)}=1$ and $a_{A C}^{(1)}=0$. Node $\mathrm{C}$ liquidates $m_{A C}=10$ shares of collateral, but node $\mathrm{B}$ liquidates only 2 shares and returns 3 to node $\mathrm{A}$. The total first-round payments are $q_{A B}^{(1)}=q_{A C}^{(1)}=10$, and there are no second-round payments. However, the total amount of collateral liquidated has been reduced from 15 to 12 .

As in this example, the analysis of Appendix A.7 shows that when collateral is held in cash-like assets, the spread of losses through the network is unaffected by the order of collateral seizure and partial payments. However, as the example suggests, the collateral-first protocol results in greater collateral liquidation. As a consequence, the collateral-first protocol can result in greater losses when collateral is illiquid.

If we interpret the payments-first protocol as the result of an automatic stay on collateral seizure by the counterparties to a failed node, then this observation is in line with policy recommendations of Duffie and Skeel [15] and the subsequent finalized stay rule on collateral sale in repo markets. The stay rule allows immediate seizure and liquidation of collateral only if it is held in cash or cash-like assets.

\section{Accelerated Payment Obligations from Contract Termination}

A financial firm's failure to make a payment due on one contract may trigger the termination of other contracts on which no payments are due. This is particularly true in over-the-counter 
derivatives markets. OTC derivative contracts often provide participants the right to terminate a contract if the counterparty enters bankruptcy, even if the counterparty has met all obligations under the contract. Bankruptcy courts also provide failed firms certain rights to terminate contracts. Upon termination, the market value of a swap or other derivative contract becomes due from the out-of-the-money party to the in-the-money party; in this sense, a default can accelerate payment obligations that would not otherwise be due.

In this section, we augment the model of Section 3 to incorporate this feature. We will show that accelerated payments from contract termination can create inconsistencies in a network model similar to those we encountered with collateral. We again resolve these complications by carefully specifying the timing of events. Whereas clearing a network with collateral could require two rounds, addressing contract termination may require as many rounds as there are nodes, because each round of terminations may trigger further defaults and thus further terminations. In this section, we assume that all contracts with a node are terminated upon the node's default; in the next section, we address selective termination.

Let

$$
v_{i j}=\text { positive value to node } j \text { of its derivative contracts with node } i \text {. }
$$

Under full contract termination, the default of node $i$ triggers the termination of its derivatives; at termination, node $i$ incurs an obligation to pay node $j$ the outstanding value $v_{i j}$, if $v_{i j}>0$. If however $v_{j i}>0$, then the default of $i$ triggers a payment obligation of $v_{j i}$ from $j$ to $i$. We assume that all contracts between $i$ and $j$ are fully netted, so $v_{i j} v_{j i}=0$.

Whereas the $\bar{p}_{i j}$ represent payments due under ordinary circumstances (including, for example, routine payments on swap contracts), the $v_{i j}$ represent asset $\left(v_{j i}>0\right)$ or liability $\left(v_{i j}>0\right)$ values for node $i$ that turn into payment obligations only upon contract termination. If all derivatives were subject to daily settlement (like futures contracts) then all changes in market values would be offset by daily payments and we would always have $v_{i j}=v_{j i}=0$. In practice, the balance sheets of large banks show significant derivatives assets and liabilities, indicating that not all contracts are settled daily.

The contingent payment obligations created by contract termination create some of the same complications we saw previously, as the following example illustrates:

Example 4.1. Consider a three-node network with

$$
\bar{p}_{12}=4, \bar{p}_{13}=2, \bar{p}_{32}=2, c_{1}=c_{2}=4 \text {. }
$$

All other model parameters are zero, except for the termination values, which we specify shortly. Node 1 defaults as its cash, $c_{1}=4$, falls below its required payments to nodes 2 and 3 . Node 
1 pays $p_{12}=a_{12} c_{1}=4 / 6 \times 4 \approx 2.67$ to node 2 , and $p_{13}=2 / 6 \times 4 \approx 1.33$ to node 3 . Node 3 defaults as the payment of $p_{32}=1.33$ it makes to node 2 is less than $\bar{p}_{32}=2$. Prior to contract termination, node 2 does not have any payment obligations.

Suppose $v_{21}=2$ and $v_{13}=v_{23}=0$. Node 2 , with cash of $c_{2}+p_{12}+p_{32}=8$, pays $v_{21}=2$ to node 1 . With this influx of cash, node 1 can now fully meet its payment obligations to nodes 2 and 3. In other words, node 1 can make all payments due, apparently avoiding default, but only if it defaults! This example shows that it is not always possible to simultaneously specify a consistent set of payments and default designations with automatic contract termination, even without collateral.

To resolve this type of inconsistency, we separate payments into rounds as we did before, consistent with the sequence of events described informally in Example 4.1. However, each round of contract terminations can now potentially trigger additional payment obligations and therefore additional defaults. In a network with $N$ nodes, we may have up to $N$ rounds of defaults and $N$ rounds of payments.

As in Section 3.3, we include the sale of any collateral in the total payment from node $i$ to node $j$ and denote this total payment by $p_{i j}$. We assume the network follows the collateral-first protocol of Sections 3.1 and 3.2. Perhaps most importantly, was assume that if node $i$ defaults in Round $\ell$, then any termination values $v_{i j}$ or $v_{j i}$ triggered by this default become payment obligations in Round $\ell+1$. This timing is consistent with the interpretation of accelerated obligations as consequences of default rather than causes of default.

\section{Round 1}

Round 1 proceeds exactly as in Section 3.1 . The first-round quantities $p_{i j}^{(1)}, \pi^{(1)}, A_{i}^{(1)}, D, a_{i j}^{(1)}$, and $\Delta^{(1)} \equiv \Delta$ are defined by equations $17,-22$.

\section{Subsequent Rounds}

We now consider round $m, 2 \leq m \leq N$. With $c_{i}^{(1)}=c_{i}$, the cash available to node $i$ at the beginning of the round is given by

$$
c_{i}^{(m)}=c_{i}^{(m-1)}+\sum_{k \neq i} p_{k i}^{(m-1)}-\sum_{k \neq i} p_{i k}^{(m-1)},
$$

with $\bar{p}_{i k}^{(1)}=\bar{p}_{i k}$. We will let $D^{(l)}$ denote the set of nodes that default in round $l$ (and not before) and define $S_{n}$ to be the set of nodes that survive rounds $1, \ldots, n$,

$$
S_{n}=\left\{i: i \notin \bigcup_{l=1}^{n} D^{(l)}\right\},
$$


with $1 \leq n \leq N$, and $S_{0}=\mathcal{N}$. Payment obligations in round $m$ are defined by

$$
\bar{p}_{i j}^{(m)}= \begin{cases}v_{i j}+\bar{p}_{i j}^{(m-1)}-p_{i j}^{(m-1)}, & i \text { or } j \in D^{(m-1)}, \text { and } i, j \in S_{m-2} ; \\ \bar{p}_{i j}^{(m-1)}-p_{i j}^{(m-1)}, & i \text { or } j \notin S_{m-2} ; \\ 0, & i, j \in S_{m-1} .\end{cases}
$$

In each round, any previous payment obligation $\bar{p}_{i j}^{(m-1)}$ is reduced by any payment made $p_{i j}^{(m-1)}$. A default by either node in the previous round creates the additional obligation $v_{i j}$ from contract termination. If both nodes have survived to the current round, then the original payment obligation $\bar{p}_{i j}$ was met in the first round and no subsequent obligation has been introduced, so the remaining obligation is zero.

As in Section 2.3, defaults in the previous round may free collateral in the current round. Recovered shares of collateral in round $m$ are given by

$$
r_{i}^{(m)}= \begin{cases}\sum_{j \in S_{m-2}}\left[m_{i j}-\Delta_{i j}^{(m-1)}\right], & i \in D^{(m-1)} ; \\ \sum_{j \in D^{(m-1)}} m_{i j}, & i \in S_{m-1} .\end{cases}
$$

The number of shares of collateral posted by $i$ that are seized and liquidated by $j$ in round $m$ is given by

$$
\Delta_{i j}^{(m)}= \begin{cases}m_{i j} \wedge \frac{\bar{p}_{i j}^{(m)}}{\pi^{(m)}}, & i \in D^{(m)}, \text { and } j \in S_{m-1} \\ 0, & \text { otherwise. }\end{cases}
$$

Given total payments $p_{i j}^{(m)}$ and a share price $\pi^{(m)}$, let

$$
A_{i}^{(m)}=c_{i}^{(m)}+\pi^{(m)} r_{i}^{(m)}+\sum_{k \neq i} p_{k i}^{(m)}
$$

denote the value of node $i$ 's remaining cash and seized and returned collateral. The set of nodes that default in the $m$ th round is given by

$$
D^{(m)}=\left\{i: i \in S_{m-1} \text {, and } A_{i}^{(m)}<\sum_{k \neq i} \bar{p}_{i k}^{(m)}\right\} .
$$

Set

$$
a_{i j}^{(m)}=\left[\bar{p}_{i j}^{(m)}-\pi^{(m)} \Delta_{i j}^{(m)}\right] / \sum_{k \neq i}\left[\bar{p}_{i k}^{(m)}-\pi^{(m)} \Delta_{i k}^{(m)}\right] .
$$

Clearing payments in round $m$ must satisfy

$$
p_{i j}^{(m)}=\bar{p}_{i j}^{(m)} \wedge\left[\pi^{(m)} \Delta_{i j}^{(m)}+a_{i j}^{(m)} A_{1}^{(m)}\right] .
$$


The number of shares of returned collateral liquidated by node $i$ is given by 10

$$
\Gamma_{i}^{(m)}=r_{i}^{(m)} \wedge \frac{1}{\pi^{(m)}}\left[\sum_{j \neq i} \bar{p}_{i j}^{(m)}-\left(c_{i}^{(m)}+\sum_{k \neq i} p_{k i}^{(m)}\right)\right] .
$$

With the totals

$$
\Gamma^{(m)}=\sum_{i} \Gamma_{i}^{(m)} \quad \text { and } \quad \Delta^{(m)}=\sum_{i} \sum_{j} \Delta_{i j}^{(m)}\left(p^{(m)}, \pi^{(m)}\right)
$$

the amount of collateral liquidated in round $m$ drives the price to

$$
\pi^{(m)}=G\left(\pi^{(m-1)}, \Gamma^{(m)}+\Delta^{(m)}\right)=\pi^{(m-1)} e^{-\alpha\left(\Gamma^{(m)}+\Delta^{(m)}\right)} .
$$

Proposition 4.1. For any levels of derivatives values $\left\{v_{i j}, i, j \in \mathcal{N}\right\}$, there exist clearing payments and prices $\left(p^{(m)}, \pi^{(m)}\right), m=1, \ldots, N$.

With the benefit of this result, we can revisit Example 4.1. Nodes 1 and 3 do indeed default in Round 1; their first-round payments are $p_{12}^{(1)}=8 / 3$, and $p_{13}^{(1)}=4 / 3$. The default of node 1 creates a new payment obligation $v_{21}$ for node 2 ; the second-round payments are $p_{21}^{(2)}=v_{21}=2$, $p_{12}^{(2)}=4 / 3$, and $p_{13}^{(2)}=2 / 3$. At the end of the second round, all payment obligations have been met.

\section{Bankruptcy Stays and Selective Termination}

The pros and cons of OTC derivative contract termination at bankrutpcy have been debated since the 1990s, and the matter has received renewed attention since the failure of Lehman Brothers. We provide some background before adapting our model to consider some of the key tradeoffs.

Most creditors in bankruptcy are subject to a stay that prevents them from seizing assets of a bankrupt entity. This provision is intended to improve the chances that the debtor will return to viability or to maximize the value of the debtor's assets to repay creditors. Derivatives and certain other financial contracts have long been exempt from these stays. As explained in Chapter 9 of Skeel [38, the exemption was introduced to reduce the risk of spillovers upon the failure of a financial firm by giving special protections to derivatives counterparties.

Since the failure of Lehman Brothers, regulators have come to have a different perspective, seeing termination rights as potentially destabilizing. Fleming and Sarkar [22] report that

\footnotetext{
${ }^{10}$ In formulating $\Gamma_{i}^{(m)}$, we have made the reasonable assumption that banks use their liquid assets before selling illiquid returned collateral. If the order is reversed, $\Gamma_{i}^{(m)}$ becomes $r_{i}^{(m)} \wedge \sum_{j \neq i} \bar{p}_{i j}^{(m)} / \pi^{(m)}$.
} 
Lehman's derivatives counterparties selectively terminated contracts where they stood to gain, but maintained contracts where termination would have resulted in a payment to Lehman, a practice often referred to as cherry-picking 11 The FDIC [19] reported that contract terminations by Lehman's counterparties caused market disruptions and left Lehman exposed to greater market risk. In 2017, the Federal Reserve [20] adopted rules placing some limits on termination of derivatives or, more precisely, qualified financial contracts (QFCs). In explaining the need for an automatic stay on terminations, the Federal Reserve [20], p.42883, noted the risk of a "chain reaction" of failures and the risk of fire sales from the liquidation of large volumes of collateral assets 12

To capture the phenomenon of creditor cherry-picking — which we refer to as selective termination - we modify the framework of Section 4 . We assume that upon the failure of node $i$, all amounts $v_{i j}$ become due, but amounts $v_{j i}$ do not come due (unless node $j$ also defaults). In other words, if node $j$ survives when node $i$ fails, node $j$ terminates contracts that trigger obligations from node $i$, but not contracts that trigger obligations to node $i$. We will compare defaults and payment shortfalls under full termination and selective termination ${ }^{13}$

\subsection{Full Versus Selective Contract Termination}

We detailed the full termination model in Section 4. In contrasting the two scenarios, we will use the following notation for the full termination (FT) and selective termination (ST) models: ${ }^{14}$

$p_{i j}^{(\ell)}, \bar{p}_{i j}^{(\ell)}, \pi^{(\ell)}$ : payments, payment obligations, and collateral prices in the FT model;

$q_{i j}^{(\ell)}, \bar{q}_{i j}^{(\ell)}, \hat{\pi}^{(\ell)}$ : payments, payment obligations, and collateral prices in the ST model;

\footnotetext{
${ }^{11}$ As explained in Skeel and Jackson [39, debtors may engage in their own form of cherry-picking. With the protection of a bankruptcy stay, a debtor may decide which contracts to "assume" and which to "reject." Skeel and Jackson [39] also explain that this optionality is consistent with the treatment of "executory" contracts in non-financial bankruptcies.

${ }^{12}$ Title II of the Dodd-Frank Act and the resolution framework of FDIC (in coordination with the Federal Reserve and OCC) impose a 1-2 day stay for QFC counterparties of the most complex U.S. bank holding companies (U.S. G-SIBs). Under the U.S. Treasury's proposed chapter 14 bankruptcy process, termination rights of QFC counterparties will be stayed for 2 days (U.S. Treasury Report [4]). That is, automatic stays for QFCs can be in place both in bankruptcy and in resolutions proceedings.

${ }^{13}$ Although we interpret selective termination as the result of creditor cherry-picking, a similar outcome would result if the failed node chose to terminate all its out-of-the-money contracts. This possibility is noted in Skeel and Jackson [39], p.181. By terminating these contracts, a failed node could force its counterparties to accept lower payments through bankruptcy proceedings.

${ }^{14}$ Our use of $q$ and $\hat{\pi}$ in this section should not be confused with their use in Section 3.3 In both cases, we use $q$ and $\hat{\pi}$ to indicate an alternative to a model that uses $p$ and $\pi$.
} 
The evolution of the ST model is identical to that of the FT model with one exception: in place of (26), the payment obligations become

$$
\bar{q}_{i j}^{(m)}= \begin{cases}v_{i j}+\bar{q}_{i j}^{(m-1)}-q_{i j}^{(m-1)}, & i \in D^{(m-1)}, \text { and } i, j \in S_{m-2} ; \\ \bar{q}_{i j}^{(m-1)}-q_{i j}^{(m-1)}, & i \text { or } j \notin S_{m-2} \\ 0, & i, j \in S_{m-1} .\end{cases}
$$

Only the first case has changed: the obligation $v_{i j}$ is added only if $i$ defaults.

By construction, the FT and ST models are identical in Round 1, so they admit the same sets of clearing payments $p_{i j}^{(1)}$ and $q_{i j}^{(1)}$. Moreover, under selective termination, a node that survives Round 1 has no payment obligations in Round 2, so no defaults occur after Round 1, and the process terminates at the end of Round 2. By the argument used for Proposition 4.1. we have the following result:

Proposition 5.1. For any levels of derivatives values $\left\{v_{i j}, i, j \in \mathcal{N}\right\}$, there exist clearing payments and prices $\left(q^{(1)}, \hat{\pi}^{(1)}\right)$ and $\left(q^{(2)}, \hat{\pi}^{(2)}\right)$. The feasible clearing payments and collateral prices in Round 1 under the ST and FT models coincide, and thus so do the first round payment shortfalls. The ST default set is a subset of the FT default set.

Because the two models agree in the first round, they produce the same defaults in Round 1; and because the ST model has no subsequent defaults, full termination always produces at least as many defaults as selective termination. The comparison of payment shortfalls is less clear. Full termination creates additional payment obligations and thus more opportunities for payments to fall short; but full termination can also increase the flow of payments, potentially offsetting the first effect.

To formulate the comparison precisely, we use the notation in $(29)$ to denote the total default sets for the two models (full and selective termination) by

$$
D^{f}=\bigcup_{l=1}^{m} D^{(l)}, D^{s}=D^{(1)}
$$

We define payment shortfalls for the two models as differences between payments due and payments made,

$$
L^{f}=\sum_{i \in D^{f}}\left(v_{i}+\bar{p}_{i}-p_{i}\right), \text { and } L^{s}=\sum_{i \in D^{s}}\left(v_{i}+\bar{p}_{i}-q_{i}\right)
$$

where $p_{i}=\sum_{l=1}^{m} p_{i}^{(l)}, q_{i}=q_{i}^{(1)}+q_{i}^{(2)}$, and $v_{i}=\sum_{j \neq i} v_{i j}$. These shortfall measures are calculated relative to payments currently due — which is the relevant focus in a moment of market stress 


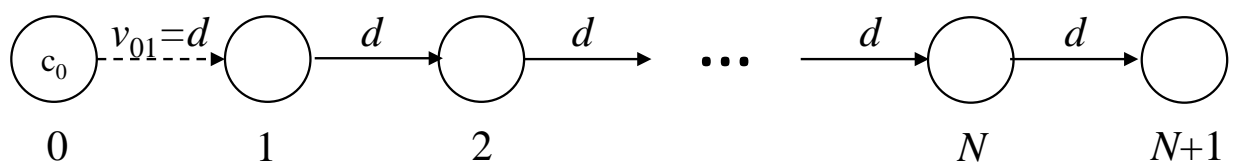

Figure 7: Network of Example 5.1. The dashed-line payment from node 0 to node 1 becomes due only upon contract termination. Nodes $1, \ldots, N+1$ have no cash.

or a crisis following defaults - and ignore future obligations. The total payments due in the FT case are always at least as large as in the ST case, but the comparison of shortfalls can go either way, as the following example illustrates.

Example 5.1. Consider the linear network of Figure 7 with nodes labeled $0,1, \ldots, N+1$. Nodes $i=1, \ldots, N$ have payments due to their successor nodes, $\bar{p}_{i, i+1}=d$. Node 0 has a potential obligation $v_{01}=d$. Node 0 holds $c_{0}$ in cash; no other nodes have cash. In Round 1 , nodes $1, \ldots, N$ default. Under selective termination, nothing more happens; none of the $N d$ payments due are made, so $L^{s}=N d$.

Under full termination, the default of node 1 triggers termination of the contract between 0 and 1 , creating a payment obligation $\bar{p}_{01}^{(2)}=v_{01}=d$. Node 0 pays $\left(d \wedge c_{0}\right)$ to node 1 , and this amount is passed through all downstream nodes. The payment shortfall becomes $L^{f}=(N+1) d-(N+1)\left(d \wedge c_{0}\right)=(N+1)\left(d-c_{0}\right)^{+}$. By varing the parameters $N$ and $d$, we can make $L^{f}=0$ and $L^{s}$ arbitrarily large, but we can also make $L^{f}-L^{s}$ arbitrarily large.

This example suggests the following properties: If full termination does not increase the set of defaults, then it (weakly) lowers the payment shortfall, compared with selective termination. Unless a node is exactly on the boundary of default, a sufficiently small increase in payment obligations will not push it into default. Thus, for sufficiently small $v_{i j}$, we have $L^{f} \leq L^{s}$; but by increasing some $v_{i j}$ we can make $L^{f}-L^{s}$ arbitrarily large, so long as $j$ defaults and $i$ does not. The key then to comparing payment shortfalls is to understand the magnitudes of derivatives liabilities relative to node distances from their default boundaries. We interpret these relative magnitudes as measures of derivatives leverage.

As a first step in formalizing these ideas, we show that full termination increases payments when collateral value is constant. Recall that the ST and FT models coincide in Round 1.

Lemma 5.1. Suppose the collateral price is constant $\pi=\hat{\pi}=1$. Then full termination and selective termination models satisfy

$$
p_{i j}^{(2)} \geq q_{i j}^{(2)}, \text { for all } i, j \in \mathcal{N}
$$


taking the smallest or largest clearing payments under each model.

In the proof of the lemma, we confirm that the smallest and largest clearing payments are well-defined for the two models. We compare these extremal solutions to account for the possibility of non-uniqueness. If each model has unique second-round clearing payments, then (36) holds directly. In light of these considerations we will compare models under payments satisfying either of the following conditions:

$$
\begin{gathered}
p^{(1)}=q^{(1)}, \pi^{(1)}=\hat{\pi}^{(1)} \text {, and } p^{(2)} \text { and } q^{(2)} \text { are the largest second round payments; } \\
p^{(1)}=q^{(1)}, \pi^{(1)}=\hat{\pi}^{(1)} \text {, and } p^{(2)} \text { and } q^{(2)} \text { are the smallest second round payments. }
\end{gathered}
$$

When the ST and FT models produce the same default sets, the additional termination obligations under the FT model must be fully met - otherwise, they would trigger additional defaults. Combining this observation with Lemma 5.1 yields the following.

Corollary 5.1. Suppose either (37) or (38) holds and the collateral price is constant. If $D^{f}=$ $D^{s}$ then $L^{f} \leq L^{s}$.

To build on this observation, we develop the notion of derivatives leverage introduced informally above. Let $e_{i}$ denote the net worth of node $i$ at the end of Round 1 ,

$$
e_{i}=c_{i}+\pi^{(1)} r_{i}+\sum_{k \neq i} p_{k i}^{(1)}-\sum_{k \neq i} \bar{p}_{i k}
$$

Proposition 5.2. Suppose (37) or (38) holds. Full termination reduces payment shortfalls, in the sense that $L^{f} \leq L^{s}$, under the "aggregate derivatives leverage" condition

$$
\sum_{i \in D^{f}-D^{(1)}} v_{i} \leq \sum_{i \in D^{f}-D^{(1)}} e_{i}
$$

if either (i) the collateral price is constant $\pi=\hat{\pi}=1$, or (ii) there is no excess collateral, meaning that $m_{i j} \leq \bar{p}_{i j}, \forall i, j \in \mathcal{N}$.

We call (40) a derivatives leverage condition because it compares derivatives liabilities on the left with a measure of equity on the right. A simple sufficient condition for $(40)$ is $v_{i} \leq e_{i}$, for all $i \notin D^{(1)}$. This condition applies to Example 5.1. Only node 0 survives the first round, and its net worth is $e_{0}=c_{0}$. If $c_{0} \geq v_{0}=d$, then $L^{f}=0$ and thus $L^{f} \leq L^{s}$. Condition 40 also holds when $D^{f}=D^{(1)}$, the case considered in Corollary 5.1 .

To add some qualitative context to (40), we note that post-crisis capital and liquidity regulations have significantly increased bank capital and liquidity levels. According to the U.S 
Department of the Treasury Report [42], p.37, large U.S. banks hold nearly $24 \%$ of their assets in high-quality liquid assets such as cash and U.S. Treasury securities. We know from bank regulatory reporting that derivatives transactions constitute a small portion of the balance sheets of even the largest U.S. bank holding companies. These considerations suggest that the cash demands from contract terminations are unlikely to topple an otherwise solvent bank, favoring full termination over selective termination.

In defining the shortfall measure $L^{s}$ in (35), we have not included contracts that have positive value $v_{k i}$ for a failed node $i \in D^{s}$, where $k \notin D^{s}$ is a surviving node: under selective termination, these contracts are not terminated and the payment obligations are not accelerated. To include these quantities as payment shortfalls, we can define

$$
L^{s+}=L^{s}+\sum_{k \notin D^{s}} \sum_{i \in D^{s}} v_{k i}
$$

As $L^{s} \leq L^{s+}$, we clearly have $L^{f} \leq L^{s+}$ under the conditions of Proposition 5.2 .

The following modification of Example 5.1 highlights the effect of collateral fire sales on the total payment shortfalls under full and selective termination.

Example 5.2. We modify the linear network of Example 5.1 by setting $c_{0}=0$ to remove node 0 's cash and introducing collateral shares $m_{01}>0$ posted by node 0 to node 1 . With a fixed collateral price $\pi \equiv 1$ and $m_{01}=c_{0}$, the shortfalls in this network are identical to those in Example 5.1: following the first-round default of nodes $1, \ldots, N$, the collateral $m_{01}$ is returned to node 0 , and node 0 pays $d \wedge m_{01}$ to node 1 in the case of full termination; it pays nothing in the case of selective termination.

Suppose $m_{01}>d$, so that node 0 could meet its obligation in the FT case if $\pi \equiv 1$, resulting in $L^{f}=0$. If the collateral is illiquid, its value will drop when node 0 sells collateral shares. Applying (31) and 32 , we find that the amount liquidated is $\Gamma^{(2)}=m_{01} \wedge d / \pi^{(2)}$, with $\pi^{(2)}=\exp \left(-\alpha \Gamma^{(2)}\right)$, so $\Gamma^{(2)}=m_{01} \wedge d \exp \left(\alpha \Gamma^{(2)}\right)$. If $m_{01}<d \exp \left(\alpha m_{01}\right)$, then $\Gamma^{(2)}=m_{01}($ all shares are liquidated) and the amount node 0 pays to node 1 is $\pi^{(2)} m_{01}=m_{01} \exp \left(-\alpha m_{01}\right)<d$. The resulting shortfall is $L^{f}=(N+1)\left(d-m_{01} \exp \left(-\alpha m_{01}\right)\right)^{+}$, whereas $L^{s}=N d$. We thus have $L^{f} \geq L^{s}$ for sufficiently large $\alpha$. In other words, the illiquidity of the collateral can reverse the order of the two shortfalls.

\subsection{Comparison with No Termination}

If we set aside the option for a bankrupt node to reject certain contracts, we can model an automatic stay by supposing that no payments are accelerated at default. This no-termination 
scenario is equivalent to setting all $v_{i j}=0$, which reduces to the model of Sections 2 and 3 . We compare no termination with full termination and selective termination.

We begin with the comparison between full termination and no termination. We continue to use $p_{i j}$ to denote clearing payments under the full termination protocol; in this section, we use $q_{i j}$ to denote clearing payments with no accelerated payments. The two scenarios coincide in Round 1; and without contract terminations no defaults occur after Round 1, and no payments are made after Round 2. We compare payments under the assumption that (37) or (38) holds, applying these conditions to the new $q_{i j}$. The total payment shortfall in the no-termination scenario is given by

$$
L^{n}=\sum_{i \in D^{(1)}}\left(\bar{p}_{i}-q_{i}\right)
$$

with $q_{i}=q_{i}^{(1)}+q_{i}^{(2)}$.

Proposition 5.3. Suppose that (37) or (38) holds. Suppose there is no excess collateral, meaning that $m_{i j} \leq \bar{p}_{i j}, \forall i, j \in \mathcal{N}$. Then full termination results in the same default set as no termination and lower payment shortfalls, $L^{f} \leq L^{n}$, if the following condition holds:

$$
v_{i} \leq \begin{cases}e_{i}, & i \notin D^{(1)} ; \\ \sum_{k \notin D^{(1)}} v_{k i}, & i \in D^{(1)} .\end{cases}
$$

The comparison of full termination and no termination presents tradeoffs similar to those in Section 5.1. Contract termination creates additional payment obligations, but it can also increase the flow of cash to meet payment obligations. As in Proposition 5.2, the condition in (41) can be interpreted as a constraint on derivatives leverage.

If we take the view that counterparties of a failed node will have to replace their contracts, then we may define

$$
L^{n+}=\sum_{i \in D^{(1)}}\left(v_{i}+\bar{p}_{i}-\bar{q}_{i}\right) .
$$

This shortfall measure includes the total value $v_{i}$ of node $i$ 's outstanding contracts and thus the replacement cost imposed on node $i$ 's counterparties. Proposition 5.3 clearly applies with $L^{n}$ replaced by $L^{n+}$.

For the comparison of selective termination (creditor cherry-picking) and no termination, we have the following simpler result. Recall that the two models coincide in Round 1.

Proposition 5.4. Suppose there is no excess collateral, meaning that $m_{i j} \leq \bar{p}_{i j}, \forall i, j \in \mathcal{N}$. For any common set of first-round payments under selective termination and no termination, we have $D^{n}=D^{s}$ and $L^{n} \leq L^{s}$. 
The network is unlikely to have excess collateral following a large shock. But if we drop the assumption of no excess collateral, the comparison of shortfalls could go either way. For example, suppose in Figure 7 that node 0 has a payment obligation to some other node $\mathrm{A}$ that it cannot meet, and suppose node 0 has posted excess collateral to node A. That excess collateral is returned to node 0 in Round 2. If node 1 selectively terminates its contract with node 0 , this creates a new payment obligation $v_{01}$, potentially increasing the systemwide shortfall. But if the excess collateral returned to node 0 is large, the contract termination will lead to additional payments by nodes 1 through $N$, potentially reducing the systemwide shortfall.

\section{Concluding Remarks}

This paper introduces a framework to study contagion in collateralized financial networks and to analyze the effects of the contract termination rules that control access to collateral. We compare alternative scenarios through their impact on the set of nodes that default and the total payment shortfall. In a collateralized network, the failure of one firm may improve the ability of other firms to meet their obligations. We show that this phenomenon makes the problem of determining clearing payments ill-posed. We resolve this difficulty and arrive at a well-defined set of clearing payments by carefully specifying the timing of payments and collateral liquidation.

It is interesting to view our analysis through a regulatory lens. In its comparison of margin requirements and capital requirements, BCBS-IOSCO [4] (p.4) writes that, margin can be seen as offering enhanced protection [in comparison with capital] against counterparty credit risk provided that it is effectively implemented. In order for margin to act as an effective risk mitigant, it must be (i) accessible when needed and (ii) provided in a form that can be liquidated rapidly and at a predictable price even in a time of financial stress. Our analysis of fire sales reinforces the second point, and our baseline model of collateral presupposes that collateral is accessible at default. But our results also show that even when (i) and (ii) hold, collateral is not guaranteed to improve financial stability. Depending on how collateral is allocated to counterparties, it can increase or decrease defaults and payment shortfalls. For instance, we show that committing excess collateral may increase risks to financial stability. This result is applicable with derivative contracts where initial margin can lead to collateral levels in excess of current payment obligations. Moreover, a comparison of alternative policies on collateral seizure requires consideration of a firm's positions in a network of payment obligations and cannot be made by considering a firm in isolation.

The same point - that the network matters - applies to the comparison of alternative 
rules on contract termination. The debate over stays on contract termination upon the failure of a firm gained renewed attention after the failure of Lehman Brothers. Policies adopted earlier argued that financial stability required protecting the termination rights of surviving counterparties; more recently, the regulatory consensus has argued that financial stability requires limiting these rights. Our analysis compares systemwide losses and defaults under alternative assumptions about contract termination. These comparisons require analyzing the network; our results and examples show that none of the termination scenarios we consider is uniformly better than the others in stemming losses.

We are able to make a stronger statement under a constraint on derivatives leverage in the network. When this condition holds, full termination results in lower payment shortfalls than selective termination or no termination. A reduction in derivatives leverage is consistent with post-crisis increases in bank capital and a general decline in over-the-counter derivatives, adding to the relevance of the condition we introduce.

\section{Acknowledgments}

For discussions and comments, we thank Robert Anderson and Darrell Duffie, as well as seminar participants at the European Central Bank, Bank for International Settlements, Bank of England, Columbia University, New York University, UC Berkeley, University of Oxford, International Monetary Fund, 2019 and 2020 QuantMinds International, and 2019 SIAM Conference on Financial Mathematics and Engineering.

\section{A Appendix: Supporting Analysis}

\section{A.1 Proof of Lemma 2.1}

Write $\mathcal{S}_{i}=\left\{k: p_{i k}^{(1)}<\bar{p}_{i k}\right\}$ for the set of nodes to which node $i$ did not meet its payment obligations in Round 1. If $j \notin \mathcal{S}_{i}$ then $a_{i j}^{(2)}=0$, so we may suppose $j \in \mathcal{S}_{i}$; in particular, $\mathcal{S}_{i}$ is nonempty. The sums in the denominators of $a_{i j}^{(1)}$ and $a_{i j}^{(2)}$ can be restricted to $k \in \mathcal{S}_{i}$. We prove the result in the more general setting of Section 3 , for which $\pi^{(1)}=\pi^{(2)}=1$ is a special case. For all $k \in \mathcal{S}_{i}$,

$$
p_{i k}^{(1)}=\pi^{(1)} m_{i k}+a_{i k}^{(1)} A_{i}^{(1)} .
$$


Making this substitution in 11 and 13 and letting $K_{i}$ denote the denominator of $a_{i j}^{(1)}$ yields

$$
\begin{aligned}
a_{i j}^{(2)} & =\frac{\bar{p}_{i j}-\pi^{(1)} m_{i j}-a_{i j}^{(1)} A_{i}^{(1)}}{\sum_{k \in \mathcal{S}_{i}}\left(\bar{p}_{i k}-\pi^{(1)} m_{i k}-a_{i k}^{(1)} A_{i}^{(1)}\right)} \\
& =\frac{\left(\bar{p}_{i j}-\pi^{(1)} m_{i j}\right)\left(1-A_{i}^{(1)} / K_{i}\right)}{\sum_{k \in \mathcal{S}_{i}}\left(\bar{p}_{i k}-\pi^{(1)} m_{i k}\right)\left(1-A_{i}^{(1)} / K_{i}\right)} \\
& =a_{i j}^{(1)} .
\end{aligned}
$$

\section{A.2 Proof of Proposition 2.1}

The existence of clearing payments in this setting is a special case of the more general claim in Proposition 3.1, which we prove separately. Here we prove (15).

The second-round payments in 14 satisfy $p_{i j}^{(2)} \leq \bar{p}_{i j}^{(2)}=\bar{p}_{i j}-p_{i j}^{(1)}$, using 11 , so $p_{i j}^{(1)}+p_{i j}^{(2)} \leq$ $\bar{p}_{i j}$. Summing over $j$, we get

$$
p_{i}^{(1)}+p_{i}^{(2)} \leq \bar{p}_{i}
$$

We will next show that the second term in 15 is also an upper bound,

$$
p_{i}^{(1)}+p_{i}^{(2)} \leq c_{i}+\sum_{k \neq i}\left(p_{k i}^{(1)}+p_{k i}^{(2)}\right)+\sum_{k \neq i} m_{i k}
$$

The second-round payments in (14) have exactly the structure of Eisenberg-Noe [17] clearing payments (4), with $r_{i}$ in (14) playing the role of $c_{i}$ in (4) and payment obligations given by $\bar{p}_{i j}^{(2)}$. Any node that did not default in the first round has no payment obligations in the second round. As in Eisenberg-Noe [17], we may write the node totals as

$$
p_{i}^{(2)}=\bar{p}_{i}^{(2)} \wedge\left(r_{i}+\sum_{k \neq i} p_{k i}^{(2)}\right) .
$$

In other words, the minimum in (14) is either attained by the first term for all $j$ or the second term for all $j$. For $i \in D$, we can rewrite $r_{i}$ using (5) and $(12)$ as $r_{i}=\sum_{k}\left(m_{i k}-\bar{p}_{i}\right)^{+}$. Making this substitution and using (11), we get

$$
p_{i}^{(2)}=\left[\bar{p}_{i}-p_{i}^{(1)}\right] \wedge\left(\sum_{k \neq i}\left(m_{i k}-\bar{p}_{i k}\right)^{+}+\sum_{k \neq i} p_{k i}^{(2)}\right) .
$$

From 100 we have

$$
p_{i k}^{(1)}=\left(m_{i k} \wedge \bar{p}_{i k}\right)+\left(a_{i k}^{(1)} A_{i}^{(1)} \wedge \bar{p}_{i k}\right) \leq\left(m_{i k} \wedge \bar{p}_{i k}\right)+a_{i k}^{(1)} A_{i}^{(1)}
$$


Summing both sides of 45 over $k$ and recalling the definition of $A_{i}^{(1)}$ in (7), we get

$$
p_{i}^{(1)} \leq c_{i}+\sum_{k \neq i} p_{k i}^{(1)}+\sum_{k \neq i}\left(m_{i k} \wedge \bar{p}_{i k}\right) .
$$

Adding (46) to (44) yields (43).

In light of $(42)$ and $(43)$, to prove $(15)$, we need to show that

$$
\text { if } p_{i}^{(1)}+p_{i}^{(2)}<\bar{p}_{i} \text { then } p_{i}^{(1)}+p_{i}^{(2)}=c_{i}+\sum_{k}\left(p_{k i}^{(1)}+p_{k i}^{(2)}\right)+\sum_{k} m_{i k} \text {. }
$$

We claim that if $p_{i}^{(1)}+p_{i}^{(2)}<\bar{p}_{i}$ then equality holds in 45 and 10 can be written as

$$
p_{i k}^{(1)}=\left(m_{i k} \wedge \bar{p}_{i k}\right)+a_{i k}^{(1)} A_{i}^{(1)} .
$$

To show this equivalence, we need to consider three cases. (i) If $\bar{p}_{i k} \leq m_{i k}$, then (6) yields $a_{i k}^{(1)}=0$, so 10 and 48 both give $p_{i k}^{(1)}=\bar{p}_{i k}$. (ii) If $\bar{p}_{i k} \geq m_{i k}+a_{i k}^{(1)} A_{i}^{(1)}$, then 10 and (48) both give $p_{i k}^{(1)}=m_{i k}+a_{i k}^{(1)} A_{i}^{(1)}$. (iii) The remaining case is $m_{i k}<\bar{p}_{i k}<m_{i k}+a_{i k}^{(1)} A_{i}^{(1)}$, which is equivalent to $0<\bar{p}_{i k}-m_{i k}<a_{i k}^{(1)} A_{i}^{(1)}$. In light of the definition of $a_{i k}^{(1)}$ in $(6)$, this implies $\sum_{j}\left[\bar{p}_{i j}-m_{i j}\right]^{+}<A_{i}^{(1)}$. But this inequality says that following the seizure of collateral, node $i$ has sufficient remaining assets to meet all its residual claims, making $p_{i k}^{(1)}=\bar{p}_{i k}$, for all $k$. Summing over $k$ yields $p_{i}^{(1)}=\bar{p}_{i}$. Thus, under the condition $p_{i}^{(1)}+p_{i}^{(2)}<\bar{p}_{i}$, case (iii) is precluded and (48) holds.

Summing over $k$ in 48 we get equality in 46). Under the condition $p_{i}^{(1)}+p_{i}^{(2)}<\bar{p}_{i}$ in (47), the minimum in (44) is attained by the second term. Adding this term to the right side of 46) yields the claimed result in (47).

\section{A.3 Analysis of Free-Termination Model}

We begin with a precise formulation of the model of Section 2.5. To capture the nodes' access to collateral, we need to set

$$
A_{i}=c_{i}+\sum_{k \neq i} p_{k i}+\sum_{k \neq i}\left[m_{i k}-\bar{p}_{i k}\right]^{+} \equiv c_{i}^{p}+\sum_{k \neq i} p_{k i}
$$

The term $\left[m_{i k}-\bar{p}_{i k}\right]^{+}$reflects excess collateral node $i$ can call back from $k$ to make payments to other nodes. Node $i$ defaults if $A_{i}<\sum_{k}\left[\bar{p}_{i k}-m_{i k}\right]^{+}$; in other words, default is the failure to meet uncollateralized obligations. We can write the default set as

$$
D^{p}=\left\{i: c_{i}+\sum_{k} p_{k i}+\sum_{k} m_{i k}<\sum_{k} \bar{p}_{i k}\right\}
$$


Upon $i$ 's default, node $j$ 's share of any remaining assets is proportional to its residual claim, so

$$
a_{i j}=\frac{\left[\bar{p}_{i j}-m_{i j}\right]^{+}}{\sum_{k}\left[\bar{p}_{i k}-m_{i k}\right]^{+}} \text {. }
$$

Clearing payments are required to satisfy

$$
p_{i j}=\bar{p}_{i j} \wedge\left[m_{i j}+a_{i j} A_{i}\right]
$$

Existence of (largest and smallest) clearing payments follows from Tarski's fixed-point theorem.

To formulate the equivalent Eisenberg-Noe model, define reduced obligations

$$
\bar{q}_{i j}=\left[\bar{p}_{i j}-m_{i j}\right]^{+}
$$

and increased cash

$$
c_{i}^{q}=c_{i}+\sum_{k}\left[m_{i k}-\bar{p}_{i k}\right]^{+}+\sum_{k}\left(m_{k i} \wedge \bar{p}_{k i}\right) .
$$

The additional cash reflects collateral $\left[m_{i k}-\bar{p}_{i k}\right]^{+}$recovered by $i$ and any paying down of obligations to $i$ using collateral posted by $k, m_{k i} \wedge \bar{p}_{k i}$. Set

$$
A_{i}^{q}=c_{i}^{q}+\sum_{k \neq i} q_{k i}
$$

and notice that $a_{i j}$ in (51) equals $\bar{q}_{i j} / \sum_{k} \bar{q}_{i k}$. With no collateral, the standard Eisenberg-Noe condition for clearing payments becomes

$$
q_{i j}=\bar{q}_{i j} \wedge a_{i j} A_{i}^{q}
$$

We may rephrase the first statement of Lemma 2.2 as saying that payments $q_{i j}$ satisfy (54) if and only if payments $p_{i j}=q_{i j}+\left(m_{i j} \wedge \bar{p}_{i j}\right)$ satisfy (52).

Proof of Lemma 2.2. If (54) holds, the substitutions $q_{i j}=p_{i j}-\left(m_{i j} \wedge \bar{p}_{i j}\right)$ and (53) yield

$$
p_{i j}-\left(m_{i j} \wedge \bar{p}_{i j}\right)=\left[\bar{p}_{i j}-m_{i j}\right]^{+} \wedge a_{i j} A_{i}^{q},
$$

so

$$
\begin{aligned}
p_{i j} & =\bar{p}_{i j} \wedge\left[\left(m_{i j} \wedge \bar{p}_{i j}\right)+a_{i j} A_{i}^{q}\right] \\
& =\bar{p}_{i j} \wedge\left[m_{i j}+a_{i j} A_{i}^{q}\right] .
\end{aligned}
$$

But

$$
\begin{aligned}
A_{i}^{q} & =c_{i}+\sum_{k}\left[m_{i k}-\bar{p}_{i k}\right]^{+}+\sum_{k}\left(m_{k i} \wedge \bar{p}_{k i}\right)+\sum_{k} q_{k i} \\
& =c_{i}+\sum_{k}\left[m_{i k}-\bar{p}_{i k}\right]^{+}+\sum_{k} p_{k i}=A_{i}
\end{aligned}
$$


so (55) yields (52). Conversely, if (52) holds, then, as $\bar{p}_{i j}=q_{i j}+\left(m_{i j} \wedge \bar{p}_{i j}\right)$, we have

$$
q_{i j}+\left(m_{i j} \wedge \bar{p}_{i j}\right)=\left[\bar{q}_{i j}+\left(m_{i j} \wedge \bar{p}_{i j}\right)\right] \wedge\left[m_{i j}+a_{i j} A_{i}^{q}\right]
$$

so

$$
q_{i j}=\bar{q}_{i j} \wedge\left[\left(m_{i j}-\bar{p}_{i j}\right)^{+}+a_{i j} A_{i}^{q}\right]=\bar{q}_{i j} \wedge a_{i j} A_{i}^{q}
$$

because if $m_{i j}>\bar{p}_{i j}$ then $\bar{q}_{i j}=0$. As $A_{i}^{q}=A_{i}$, 54 follows.

To see that the payment shortfalls coincide, observe that

$$
\bar{q}_{i j}-q_{i j}=\left[\bar{p}_{i j}-m_{i j}\right]^{+}-\left[p_{i j}-\left(m_{i j} \wedge \bar{p}_{i j}\right)\right]=\bar{p}_{i j}-p_{i j}
$$

The default sets coincide because $q_{i j}<\bar{q}_{i j}$ if and only if $p_{i j}<\bar{p}_{i j}$.

Proof of Proposition 2.3. For the model with free termination, we can use $c_{i}^{p}$ in 49 to rewrite the clearing condition $(52)$ as

$$
p_{i j}=\bar{p}_{i j} \wedge\left[m_{i j}+a_{i j}\left(c_{i}^{p}+\sum_{k} p_{k i}\right)\right] .
$$

This equation has exactly the same form as the first-round clearing payments in (10), but with $c_{i}$ in (7) replaced by $c_{i}^{p}$. As $c_{i}^{p} \geq c_{i}$, it follows from Theorem 3 of Milgrom and Roberts [33 that the largest and smallest fixed points of (56) are no smaller than, respectively, the largest and smallest fixed points of (10). In other words, payments with free termination exceed first-round payments in the original model. By comparing (8) and 50 , we see that $p_{k i} \geq p_{k i}^{(1)}$ implies $D^{p} \subseteq D$ : free termination results in fewer defaults.

To compare payment shortfalls in the two models, we use equation (57), proved below, and claim that we can replace $c_{i}+r_{i}$ with $c_{i}^{p}$ to write

$$
p_{i j}^{(1)}+p_{i j}^{(2)}=\bar{p}_{i j} \wedge\left[m_{i j}+a_{i j}^{(1)}\left(c_{i}^{p}+\sum_{k}\left(p_{k i}^{(1)}+p_{k i}^{(2)}\right)\right)\right] .
$$

If $i \notin D$, then $p_{i j}^{(1)}=\bar{p}_{i j}, p_{i j}^{(2)}=0$ and there is nothing to show. If $i \in D$, then the returned collateral is $r_{i}=\sum_{k}\left[m_{i k}-\bar{p}_{i k}\right]^{+}$, and indeed $c_{i}+r_{i}=c_{i}^{p}$. Comparison with 56 now shows that total payments in the two systems coincide.

\section{A.4 Proof of Proposition 2.4}

We first derive an expression for total payments $p_{i j}^{(1)}+p_{i j}^{(2)}$ that is of independent interest. Using Lemma 2.1. we can replace $a_{i j}^{(2)}$ in 14 with $a_{i j}^{(1)}$, because if $a_{i j}^{(2)}=0$ then $p_{i j}^{(1)}=\bar{p}_{i j}$, so $p_{i j}^{(2)}=0$ 
and is unchanged by the replacement. With this substitution and adding (10) and (14), we get

$$
\begin{aligned}
p_{i j}^{(1)}+p_{i j}^{(2)} & =\bar{p}_{i j} \wedge\left(\left[\bar{p}_{i j} \wedge\left[m_{i j}+a_{i j}^{(1)}\left(c_{i}+\sum_{k} p_{k i}^{(1)}\right)\right]+a_{i j}^{(2)}\left(r_{i}+\sum_{k} p_{k i}^{(2)}\right)\right)\right. \\
& =\bar{p}_{i j} \wedge\left[m_{i j}+a_{i j}^{(1)}\left(c_{i}+r_{i}+\sum_{k}\left(p_{k i}^{(1)}+p_{k i}^{(2)}\right)\right)\right] .
\end{aligned}
$$

This expression does not quite reduce the two rounds to a single round because the returned collateral $r_{i}$ is determined after the first round and is not an exogenous parameter. We now turn to the two claims in the proposition.

(i) Removing excess collateral has no effect on Round 1 payments and no effect on which nodes default. All excess collateral becomes returned collateral in Round 2. Replacing excess collateral with cash increases $c_{i}$ by exactly the amount it decreases returned collateral $r_{i}$, so we can see from (57) that pooling excess collateral does not affect thet set of clearing payments and therefore does not affect payment shortfalls. The increase in $c_{i}$ can, however, reduce the default set, which is determined in Round 1.

(ii) Under proportional collateral, $\left[\bar{p}_{i j}-m_{i j}\right]^{+}=\left(1-k_{i}\right) \bar{p}_{i j}$, so $a_{i j}^{(1)}=\bar{p}_{i j} / \sum_{k} \bar{p}_{i k}$, from which it follows that $m_{i j}=a_{i j}^{(1)} m_{i}$, with $m_{i}=\sum_{j} m_{i j}$. Moreover, with $k_{i} \in[0,1]$, there is no excess collateral, so no defaulting node receives any returned collateral, meaning that $a_{i j}^{(1)} r_{i}=0$. We may therefore write $(57)$ as

$$
p_{i j}^{(1)}+p_{i j}^{(2)}=\bar{p}_{i j} \wedge a_{i j}^{(1)}\left(m_{i}+c_{i}+\sum_{k}\left(p_{k i}^{(1)}+p_{k i}^{(2)}\right)\right) .
$$

As the total payments depend on $m_{i}$ and $c_{i}$ only through their sum, pooling while preserving proportional collateral (increasing $c_{i}$ by decreasing $k_{i}$ ) has no effect on payment shortfalls, but, as before, increasing $c_{i}$ can reduce defaults.

\section{A.5 Proof of Proposition 3.1}

We begin with the analysis of first-round payments. Through an arbitrary ordering of pairs of nodes, we can record the set of payments $p_{i j}^{(1)}$ in a vector $p^{(1)}$; interpret the vector $\bar{p}$ accordingly. If we take any $\pi \in[0,1]$ and $p^{(1)} \in[0, \bar{p}]$ and plug these variables into the right side of $(17)$ (22), then the variables on the left side of (17) and (22) return new values of $\pi \in[0,1]$ and $p^{(1)} \in[0, \bar{p}]$. In other words, expressions $17-22$ define a mapping $F:\left(\pi, p^{(1)}\right) \rightarrow\left(\pi, p^{(1)}\right)$ of $[0,1] \times[0, \bar{p}]$ into itself.

Lemma A.1. F is monotone increasing. 
Proof. In 22 we see that $p_{i j}^{(1)}$ is monotone increasing in $A_{i}^{(1)}$ and therefore monotone increasing in $p_{k i}^{(1)}, k \neq i$. Now consider $p_{i j}^{(1)}$ as a function of $\pi$. If $i \notin D$, then $p_{i j}^{(1)} \equiv \bar{p}_{i j}$, and changing $\pi$ has no effect on $p_{i j}^{(1)}$. For $i \in D$, we consider three cases.

Case 1. $A_{i}^{(1)}<\sum_{k}\left(\bar{p}_{i j}-\pi m_{i k}\right)^{+}$and $\bar{p}_{i k}>\pi m_{i j}$. In this case, 22, yields a right derivative of

$$
\frac{\partial p_{i j}^{(1)}}{\partial \pi}=m_{i j}-\frac{m_{i j} A_{i}^{(1)}}{\sum_{k}\left(\bar{p}_{i k}-\pi m_{i k}\right)^{+}}+\frac{\left(\bar{p}_{i j}-\pi m_{i j}\right)^{+} A_{i}^{(1)} \sum_{k} m_{i k} \mathbf{1}\left\{\bar{p}_{i k}>\pi m_{i k}\right\}}{\left.\left[\sum_{k} \bar{p}_{i k}-\pi m_{i k}\right)^{+}\right]^{2}} .
$$

The second term on the right is less than $m_{i j}$ because we have assumed $A_{i}^{(1)}<\sum_{k}\left(\bar{p}_{i k}-\pi m_{i k}\right)^{+}$. The third term is nonnegative, and the derivative is then as well. A small increase in $\pi$ yields an increase in $p_{i j}^{(1)}$.

Case 2. $A_{i}^{(1)} \geq \sum_{k}\left(\bar{p}_{i k}-\pi m_{i k}\right)^{+}$and $\bar{p}_{i k}>\pi m_{i j}$. These conditions imply $p_{i j}^{(1)}=\bar{p}_{i j}$, and they are preserved under a small increase in $\pi$, so $\partial p_{i j}^{(1)} / \partial \pi=0$.

Case 3. $\bar{p}_{i k} \leq \pi m_{i j}$. In this case, $p_{i j}^{(1)}=\bar{p}_{i j}$, so $\partial p_{i j}^{(1)} / \partial \pi=0$.

As $\pi$ increases, we may transition into Case 2 or Case 3 . Either transition yields $p_{i j}^{(1)}=\bar{p}_{i j}$, so monotonicity holds.

It remains to show that 17) makes $\pi$ on the left an increasing function of all $p_{i j}^{(1)}$ and $\pi$ on the right. Monotonicity in $\pi$ is immediate from the monotonicity of $G$. An increase in $p_{i j}^{(1)}$ leads the default set $D$ to contract or remain unchanged, resulting in a decrease in $\Delta$ and an increase in $\pi$.

By Tarski's fixed point theorem, Lemma A.1 implies that $F$ has a fixed point in $[0,1] \times[0, \bar{p}]$, which delivers the required clearing payments and collateral price. The following lemma will ensure that a fixed point is reached through iterative application of $F$, starting from the upper boundary of this domain.

Lemma A.2. $F$ is continuous from the right; that is, for any decreasing convergent sequence $\left(\pi^{\ell}, p^{\ell}\right) \rightarrow\left(\pi^{*}, p^{*}\right)$, we have $F\left(\pi^{\ell}, p^{\ell}\right) \rightarrow F\left(\pi^{*}, p^{*}\right)$.

Proof. Equations 17)-22 imply that $p_{i j}^{(1)}$ is continuous in $p^{(1)}$ and $\pi$, and 17 implies $\pi$ on the left is continuous in $\pi$ on the right. A change in $p^{(1)}$ may produce a change in $D$ and thus a discontinuity in $\pi$. However, a small increase in $p^{(1)}$ preserves the inequalities defining the default set in (18), leaving $D$ unchanged and implying right-continuity of $\pi$ in $p^{(1)}$.

We can now conclude the proof of Proposition 3.1. By Lemma A.1, the iterates of $F$ starting from $(1, \bar{p})$ form a decreasing sequence $F\left(\pi^{\ell}, p^{\ell}\right)=\left(\pi^{\ell+1}, p^{\ell+1}\right)$. This sequence is bounded is below by $(0,0)$ and therefore has a limit $\left(\pi^{*}, p^{*}\right)$. Thus, $F\left(\pi^{\ell}, p^{\ell}\right) \rightarrow\left(\pi^{*}, p^{*}\right)$. But Lemma A.2 implies that $F\left(\pi^{\ell}, p^{\ell}\right) \rightarrow F\left(\pi^{*}, p^{*}\right)$. We conclude that $F\left(\pi^{*}, p^{*}\right)=\left(\pi^{*}, p^{*}\right)$. 
This fixed point provides a first-round price and clearing vector $\left(\pi^{(1)}, p^{(1)}\right)$. The existence of $\left(\pi^{(2)}, p^{(2)}\right)$ now follows by a similar but simpler argument. Expressions 23-25) define a mapping from $\left(\pi^{(2)}, p^{(2)}\right)$ on the right to $\left(\pi^{(2)}, p^{(2)}\right)$ on the left. The mapping is clearly monotone increasing and continuous, and it maps $[0,1] \times\left[0, \bar{p}^{(2)}\right]$ into itself. It therefore has a fixed point, and the fixed point delivers the required solution $\left(\pi^{(2)}, p^{(2)}\right)$.

\section{A.6 Proof of Proposition 3.2}

The existence of a largest (and smallest) first-round solution follows from Tarski's fixed point theorem and the monotonicity of $F$ in Lemma A.1. By Theorem 3 of Milgrom and Roberts [33, the monotonicity of $F$ in $\pi^{(1)}$ implies that the largest and smallest $p^{(1)}$ are smaller with illiquid collateral $\left(\pi^{(1)} \leq 1\right)$ than with liquid collateral $\left(\pi^{(1)}=1\right)$. This implies that the default set is larger with illiquid collateral.

With illiquid collateral, the argument leading to (57) yields

$$
\begin{aligned}
p_{i j}^{(1)}+p_{i j}^{(2)} & =\bar{p}_{i j} \wedge\left[\pi^{(1)} m_{i j}+a_{i j}^{(1)}\left(c_{i}+\pi^{(2)} r_{i}+\sum_{k}\left(p_{k i}^{(1)}+p_{k i}^{(2)}\right)\right)\right] \\
& \equiv \bar{p}_{i j} \wedge\left[\pi^{(1)} m_{i j}+a_{i j}^{(1)} \tilde{A}_{i}\right] .
\end{aligned}
$$

With this representation, we may write $\left(p^{(1)}, \pi^{(1)}, p^{(1)}+p^{(2)}, \pi^{(2)}\right)=\left(F\left(p^{(1)}, \pi^{(1)}\right), \tilde{F}\left(p^{(1)}, \pi^{(1)}, p^{(1)}+\right.\right.$ $\left.p^{(2)}, \pi^{(2)}\right)$ ), with $F$ as in Lemma A.1. and $\tilde{F}$ defined by 60 and 24 .

In 60, $\tilde{A}_{i}$ is increasing in $p^{(1)}+p^{(2)}$, in $\pi^{(2)}$, and in $r_{i}$, which is increasing in $\pi^{(1)}$. Moreover, 60 has the same form as 22, so the argument in Lemma A.1 shows that $\tilde{F}$ is monotone increasing. It follows from Tarski's fixed point theorem that the mapping defined by $(F, \tilde{F})$ has a largest and smallest fixed point. By Theorem 3 of Milgrom and Roberts [33], with $\pi^{(1)}, \pi^{(2)} \leq 1$ (illiquid collateral), the largest and smallest fixed points are smaller than the largest and smallest fixed points when we set $\pi^{(1)} \equiv \pi^{(2)} \equiv 1$ (the case of liquid collateral). As illiquid collateral yields smaller total payments $p^{(1)}+p^{(2)}$, it yields larger payment shortfalls.

\section{A.7 Analysis of Collateral-First Protocol}

In several places in this paper, we compare our baseline model of Sections 2.2 2.3 and Sections 3.13 .2 with alternative models. In making these comparisons, we use the notation $q_{i j}$ and $\hat{\pi}$ (with superscripts to distinguish rounds) to denote payments and prices in the alternative model. The specific meaning of these variables will be different in different sections as we use this notation to compare our baseline model against different alternatives. 
In this section, we use $\left(q_{i j}^{(1)}, q_{i j}^{(2)}\right)$ and $\left(\hat{\pi}^{(1)}, \hat{\pi}^{(2)}\right)$ to denote two rounds of payments and collateral prices under a protocol in which payments precede collateral seizure in Round 1. First-round payments are characterized by

$$
q_{i j}^{(1)}= \begin{cases}\bar{p}_{i j} \wedge\left[a_{i j}^{(1)} A_{i}^{(1)}+\left[\left(\bar{p}_{i j}-a_{i j}^{(1)} A_{i}^{(1)}\right)^{+} \wedge \hat{\pi} m_{i j}\right]\right], & i \in D^{q} \\ \bar{p}_{i j}, & i \notin D^{q} .\end{cases}
$$

The first case should be read as follows: node $i$ makes a partial payment to node $j$ of $a_{i j}^{(1)} A_{i}^{(1)}$; any residual obligation $\left(\bar{p}_{i j}-a_{i j}^{(1)} A_{i}^{(1)}\right)^{+}$is paid from collateral, up to the amount available $\hat{\pi} m_{i j}$; the total payment cannot exceed the amount due $\bar{p}_{i j}$. The assets $A_{i}^{(1)}$ have the same form as in (7), but now with incoming payments $q_{k i}^{(1)}$; the default set is determined exactly as in (8), but we have labeled it $D^{q}$ to indicate its dependence on the payments $q^{(1)}$. The proportions $a_{i j}^{(1)}$ are as given in (19) and thus reflect the collateral posted. Upon node $i$ 's default, the shares of collateral seized and sold by node $j$ become

$$
\Delta_{i j}^{q}= \begin{cases}m_{i j} \wedge \frac{\left[\bar{p}_{i j}-a_{i j}^{(1)} A_{i}^{(1)}\right]^{+}}{\hat{\pi}^{(1)}}, & i \in D^{q} \\ 0, & i \notin D^{q}\end{cases}
$$

The first case in (62) captures the feature that a failed node's collateral can be seized and liquidated only after its other assets are exhausted. Set $\Delta^{q}=\sum_{i} \sum_{j} \Delta_{i j}^{q}$. As before, the price impact function $\hat{\pi}^{(1)}=e^{-\alpha \Delta^{q}}$ determines the equilibrium asset price. Once first-round payments and $\hat{\pi}^{(1)}$ are determined, second-round payments $q_{i j}^{(2)}$ and $\hat{\pi}^{(2)}$ are characterized by (23) - 25), just as before. The proof of Proposition 3.1 can be used to show the existence of first- and second-round clearing payments and prices $\left(q^{(1)}, \hat{\pi}^{(1)}\right)$ and $\left(q^{(2)}, \hat{\pi}^{(2)}\right)$.

In the case of liquid collateral, $\pi \equiv 1$, total payments in the original (collateral first) model and the alternative (payments first) model are the same:

Proposition A.1. Assume that collateral is posted in cash so that there are no fire-sale effects. If $\left(p_{i j}^{(1)}, p_{i j}^{(2)}\right), i, j \in \mathcal{N}$, are total clearing payments for the original model, then $q_{i j}^{(1)}=p_{i j}^{(1)}$ and $q_{i j}^{(2)}=p_{i j}^{(2)}, i, j \in \mathcal{N}$, are total clearing payments in the alternative model with delayed collateral seizure. Thus, with liquid collateral, the two protocols yield the same default set and the same payment shortfalls.

Proof of Proposition A.1. We first show that if all incoming payments to node $i$ agree under the two models, $q_{k i}^{(1)}=p_{k i}^{(1)}, k \in \mathcal{N}$, then outgoing payments $p_{i j}^{(1)}$ given by 10 and $q_{i j}^{(1)}$ given by (61) agree for all $j \in \mathcal{N}$.

If all incoming payments to node $i$ agree under the two models, then the two models yield the same $A_{i}^{(1)}$, and $i \in D$ if and only if $i \in D^{q}$. If $i \notin D$, then $q_{i j}^{(1)}=\bar{p}_{i j}=p_{i j}^{(1)}$, for all $j$. 
Suppose $i \notin D$. If $\bar{p}_{i j} \leq a_{i j}^{(1)} A_{i}^{(1)}$, then 10 and 61 both evaluate to $\bar{p}_{i j}$. If $\bar{p}_{i j}>a_{i j}^{(1)} A_{i}^{(1)}$, then (61) evaluates to

$$
q_{i j}^{(1)}=\bar{p}_{i j} \wedge\left[a_{i j}^{(1)} A_{i}^{(1)}+\left[\bar{p}_{i j}-a_{i j}^{(1)} A_{i}^{(1)}\right] \wedge m_{i j}\right]=\bar{p}_{i j} \wedge\left[\bar{p}_{i j} \wedge\left(m_{i j}+a_{i j}^{(1)} A_{i}^{(1)}\right)\right],
$$

which agrees with 10. Thus, $q_{i j}^{(1)}=p_{i j}^{(1)}$, for all $j$.

We now turn to the second-round payments. We assume that all first-round payments agree under the two models, and we assume that all second-round incoming payments agree, $q_{k i}^{(2)}=p_{k i}^{(2)}$, for all $k \in \mathcal{N}$, and we show that this implies that $q_{i j}^{(2)}=p_{i j}^{(2)}$, for all $j \in \mathcal{N}$.

If $i \notin D$, then node $i$ has no second-round payment obligations, so $q_{i j}^{(2)}=p_{i j}^{(2)}=0$, for all $j$. Suppose $i \in D$. If the amount of returned collateral $r_{i}$ is the same in the two models, then the payments made by node $i$ under the two models agree because they are determined by (14).

The only remaining case to consider is the possibility that the two models may produce different quantities of returned collateral $r_{i}$. We see from 12 that the quantities of liquidated collateral $\Delta_{i j}$ in (5) and $\Delta_{i j}^{q}$ in $(62)$ must then differ for some $j$. We always have $\Delta_{i j}^{q} \leq \Delta_{i j} \leq$ $m_{i j}$, so for the quantities to differ we must have $\Delta_{i j}^{q}<\Delta_{i j}$ and thus $\Delta_{i j}^{q}<m_{i j}$. We must then have $a_{i j}^{(1)} A_{i}^{(1)}+\Delta_{i j}^{q}=\bar{p}_{i j}$; if we had $a_{i j}^{(1)} A_{i}^{(1)}+\Delta_{i j}^{q}<\bar{p}_{i j}$, additional collateral would have been liquidated in the first round of the payments-first protocol to meet the obligation $\bar{p}_{i j}$. Using the definition of $a_{i j}^{(1)}$ in 6 , we can write the equation $a_{i j}^{(1)} A_{i}^{(1)}+\Delta_{i j}^{q}=\bar{p}_{i j}$ as

$$
\frac{\left[\bar{p}_{i j}-m_{i j}\right]^{+}}{\sum_{k \neq i}\left[\bar{p}_{i k}-m_{i k}\right]^{+}} A_{i}^{(1)}=\bar{p}_{i j}-\Delta_{i j}^{q} \Rightarrow A_{i}^{(1)}>\sum_{k \neq i}\left[\bar{p}_{i k}-m_{i k}\right]^{+} .
$$

But this inequality states node $i$ has sufficient assets to meet all its Round 1 obligations under the collateral-first protocol, so $p_{i j}^{(1)}=\bar{p}_{i j}$, hence $q_{i j}^{(1)}=\bar{p}_{i j}$, implying that $p_{i j}^{(2)}=q_{i j}^{(2)}=0$.

\section{A.8 Proof of Proposition 4.1}

The first round with contract termination is identical to the first round in Section 3.1 so the existence of $\left(p^{(1)}, \pi^{(1)}\right)$ follows from Proposition 3.1. For $m \geq 2$, we claim that $\Delta_{i j}^{(m)}=0$. In light of (27), it suffices to consider the case $i \in D^{(m)}, j \in S_{m-1}$. But if $i \in D^{(m)}$ then $i$ survived the first $m-1$ rounds, so $i \in S_{m-1}$. In this case, 26 yields $\bar{p}_{i j}^{(m)}=0$, and 27 yields $\Delta_{i j}^{(m)}=0$. With all $\Delta_{i j}^{(m)}=0$, the mapping defined by equations $26-32$ becomes a special case of the mapping in Lemma A.1, with one modification, which is the inclusion of the returned collateral value $\pi^{(m)} r_{i}^{(m)}$ in $A_{i}^{(m)}$ in 28 . The monotonicity proved in Lemma A.1 holds with this modification: the only step affected is (58), where we pick up an additional positive term from the monotonicity of $A_{i}^{(m)}$ in $\pi^{(m)}$. The existence of $\left(p^{(m)}, \pi^{(m)}\right)$ follows as in Proposition 3.1 . 


\section{A.9 Proof of Proposition 5.2}

Proof of Lemma 5.1. The second-round payment obligations in the FT model are given by

$$
\bar{p}_{i j}^{(2)}= \begin{cases}v_{i j}+\bar{p}_{i j}-p_{i j}^{(1)}, & i \text { or } j \in D^{(1)} \\ 0, & \text { otherwise. }\end{cases}
$$

This follows from (26) with $m=2$, recalling that all nodes are in $S_{0}$. Under the ST model, $q_{i j}^{(1)}=p_{i j}^{(1)}$ and the second-round payment obligations are given by

$$
\bar{q}_{i j}^{(2)}= \begin{cases}v_{i j}+\bar{p}_{i j}-p_{i j}^{(1)}, & i \in D^{(1)} \\ 0, & \text { otherwise }\end{cases}
$$

because the amount $v_{i j}$ becomes due only if $i$ defaults. Comparing (63) and (64), we can write $\bar{p}_{i j}^{(2)} \geq \bar{q}_{i j}^{(2)}, \forall i, j \in \mathcal{N}$.

Second-round payments under full termination are given by 30 with $m=2$. In the proof of Proposition 4.1. we showed that $\Delta_{i j}^{(m)}=0$ for $m \geq 2$, so 30 yields

$$
p_{i j}^{(2)}=\bar{p}_{i j}^{(2)} \wedge a_{i j}^{(2)}\left[c_{i}^{(2)}+\pi^{(2)} r_{i}^{(2)}+\sum_{k \neq i} p_{k i}^{(2)}\right], \quad a_{i j}^{(2)}=\frac{\bar{p}_{i j}^{(2)}}{\sum_{k \neq i} \bar{p}_{i k}^{(2)}},
$$

taking $a_{i j}^{(2)}=0$ if node $i$ has no second-round obligations. Under selective termination,

$$
q_{i j}^{(2)}=\bar{q}_{i j}^{(2)} \wedge \hat{a}_{i j}^{(2)}\left[c_{i}^{(2)}+\hat{\pi}^{(2)} r_{i}^{(2)}+\sum_{k \neq i} q_{k i}^{(2)}\right], \quad \hat{a}_{i j}^{(2)}=\frac{\bar{q}_{i j}^{(2)}}{\sum_{k \neq i} \bar{q}_{i k}^{(2)}} .
$$

The cash amounts $c_{i}^{(2)}$ and returned collateral $r_{i}^{(2)}$ are indeed equal in these two expressions because the two models agree in Round 1. Equations 65 and 66 represent $p^{(2)}$ and $q^{(2)}$ as fixed points of a common mapping, parameterized by $\bar{p}^{(2)}$ and $a^{(2)}$ in the first case and by $\bar{q}^{(2)}$ and $\hat{a}^{(2)}$ in the second case. Moreover, $p_{i j}^{(2)}$ in 65 is an increasing function of $\bar{p}_{i j}^{(2)}$ and $a_{i j}^{(2)}$, and $q_{i j}^{(2)}$ in 66 is an increasing function of $\bar{q}_{i j}^{(2)}$ and $\hat{a}_{i j}^{(2)}$.

We have shown that $\bar{p}^{(2)} \geq \bar{q}^{(2)}$; we now claim that $a_{i j}^{(2)} \geq \hat{a}_{i j}^{(2)}$, for all $i, j \in \mathcal{N}$. If $i \in D^{(1)}$, then we see from 63 and $\sqrt[64]{ }$ that $\bar{p}_{i k}^{(2)}=\bar{q}_{i k}^{(2)}$, for all $k$, so $a_{i j}^{(2)}=\hat{a}_{i j}^{(2)}$; and if $i \notin D^{(1)}$ then $\hat{a}_{i j}^{(2)}=0$. The ordering in 36 now follows from Theorem 3 of Milgrom and Roberts [33] for the smallest and largest fixed points of the two models, which also ensures the existences of these extremal fixed points. 
Proof of Proposition 5.2. From from (34) we have $D^{s} \subseteq D^{f}$, and as noted there $D^{s}=D^{(1)}$. To compare payment shortfalls we use 35 to write

$$
\begin{aligned}
L^{s}-L^{f} & =\sum_{i \in D^{(1)}}\left(p_{i}-q_{i}\right)-\sum_{i \in D^{f}-D^{(1)}}\left(v_{i}+\bar{p}_{i}-p_{i}\right) \\
& =\sum_{i \in D^{(1)}}\left(\sum_{\ell \geq 2} p_{i}^{(\ell)}-q_{i}^{(2)}\right)-\sum_{i \in D^{f}-D^{(1)}}\left(v_{i}+\bar{p}_{i}-p_{i}\right) \\
& =\sum_{i \in D^{(1)}}\left(\sum_{\ell \geq 2} p_{i}^{(\ell)}-q_{i}^{(2)}\right)-\sum_{i \in D^{f}-D^{(1)}}\left(v_{i}-\sum_{\ell \geq 2} p_{i}^{(\ell)}\right) .
\end{aligned}
$$

Equation 67) uses two properties: the first-round payments agree, $p_{i}^{(1)}=q_{i}^{(1)}$, for all $i$; and under selective termination, there are no payments after the second round, so $q_{i}^{(\ell)}=0$, for $\ell>2$. Equation (68) follows because if $i \notin D^{(1)}$, then node $i$ must have met its first-round payment obligations, so $\bar{p}_{i}=p_{i}^{(1)}$.

Under full termination, if node $i$ survives Round 1 but defaults in a subsequent round (i.e., $\left.i \in D^{f}-D^{(1)}\right)$ then its payments $\sum_{\ell \geq 2} p_{i}^{(\ell)}$ must be at least as large as its net worth $e_{i}$ defined in (39); if a node paid out less than its net worth, it would not default. From 68) we get

$$
L^{s}-L^{f} \geq \sum_{i \in D^{(1)}}\left(\sum_{\ell \geq 2} p_{i}^{(\ell)}-q_{i}^{(2)}\right)-\sum_{i \in D^{f}-D^{(1)}}\left(v_{i}-e_{i}\right) .
$$

In case (i), Lemma 5.1 applies, so $p_{i}^{(2)} \geq q_{i}^{(2)}$ and the first term on the right in 69 is positive. Under condition 40, we conclude from 69 that $L^{s} \geq L^{f}$. For case (ii) we note that if $m_{i j} \leq \bar{p}_{i j}$, for all $i, j \in \mathcal{N}$, then, under selective termination, nodes that default in Round 1 do not have any collateral returned and do not receive any subsequent payments, so they cannot make any subsequent payments; thus, $q_{i}^{(2)}=0$ in 69 , so 40 again implies $L^{s} \geq L^{f}$.

Proof of Proposition 5.3. Under full termination and the condition $v_{i} \leq e_{i}, \forall i \notin D^{(1)}$, a node that survives the first round can meet its second-round obligations because these obligations $v_{i}$ do not exceed the node's net worth $e_{i}$. Thus, $D^{f}=D^{(1)}$, and without further defaults, $p_{i j}^{(\ell)}=0$, for all $\ell>2$.

In the no-termination scenario, $D^{(1)}$ is also the default set, because the two models agree in Round 1. In the absence of excess collateral, a node that defaults in Round 1 has no collateral returned. If no contracts are terminated then such a node has no influx of cash and therefore cannot make any further payments: $q_{i j}^{(\ell)}=0$ for $\ell \geq 2$ if $i \in D^{(1)}$. If $i \notin D^{(1)}$, then node $i$ has no payment obligations after Round 1 so again $q_{i j}^{(\ell)}=0$ for $\ell \geq 2$. The difference in payment 
shortfalls is given by

$$
\begin{aligned}
L^{n}-L^{f} & =\sum_{i \in D^{(1)}}\left(\bar{p}_{i}-q_{i}\right)-\sum_{i \in D^{(1)}}\left(v_{i}+\bar{p}_{i}-p_{i}\right) \\
& =\sum_{i \in D^{(1)}}\left(p_{i}-q_{i}\right)-\sum_{i \in D^{(1)}} v_{i} \\
& =\sum_{i \in D^{(1)}} p_{i}^{(2)}-\sum_{i \in D^{(1)}} v_{i}
\end{aligned}
$$

where the first equation uses $D^{f}=D^{(1)}$, and the third step uses $p_{i}^{(1)}=q_{i}^{(1)}, q_{i}^{(\ell)}=0, \ell \geq 2$, and $p_{i}^{(\ell)}=0$, for all $\ell>2$.

If $i \in D^{(1)}$, then node $i$ 's total second-round payments are given by the lesser of its payment obligations and its cash influx, so

$$
\begin{aligned}
p_{i}^{(2)} & =\left(v_{i}+\bar{p}_{i}-p_{i}^{(1)}\right) \wedge\left(\sum_{k \notin D^{(1)}} v_{k i}+\sum_{k \in D^{(1)}} p_{k i}^{(2)}\right) \\
& \geq v_{i} \wedge\left(v_{i}+\sum_{k \in D^{(1)}} p_{k i}^{(2)}\right)=v_{i}
\end{aligned}
$$

using the second case in 41. It now follows from 70 that $L^{n}-L^{f} \geq 0$.

Proof of Proposition 5.4. Under both selective termination and no termination, if the network has no excess collateral then no collateral is returned in Round 2 and no payments are made in Round 2. Selective termination does not create any new defaults, so $D^{s}=D^{(1)}=D^{n}$. Compared with no termination, selective termination increases second-round payment obligations; but with no second-round payments made, this results in larger payments shortfalls, so $L^{n} \leq L^{s}$

\section{B Appendix: Institutional Features}

This appendix provides some institutional background on collateral ownership and contract termination in the markets for over-the-counter (OTC) derivatives; similar considerations apply to many forms of collateralized borrowing, including standard repurchase agreements. The points we emphasize are as follows: collateral is owned by the posting party until that party defaults; upon default by the posting party, the surviving party may seize the collateral quickly; a surviving party may face delays in recovering collateral posted to a counterparty that defaults; parties may not ordinarily terminate contracts at will as a means of recovering posted collateral.

OTC derivatives are traded either bilaterally (the non-cleared market) or through central counterparties (CCPs). Following reforms introduced in 2009, when two parties enter into a 
bilateral contract, they post collateral to each other, and the amount of collateral is updated regularly as market prices changes. The amounts exchanged may be asymmetric; for example, the seller of an option will need to post more collateral than the buyer.

These arrangements are typically governed by an ISDA (International Swaps and Derivatives Association) agreement. Under such an agreement, each party retains ownership of the collateral it pledges and grants to the other party a "priority interest" in the collateral in case the pledging party defaults. Contracts agreed to under an ISDA agreement are terminated by events of default and certain additional events including changes in law, certain tax events, force majeure events, and other triggers (such as credit downgrades) that the parties might agree to. (See, e.g., ISDA [29].) Neither party may unilaterally terminate the contract unless that option is itself part of the contract.

The internationally agreed principles governing bilateral margin (BCBS-IOSCO [4, p.23) specify that it should be "immediately available to the collecting party in the event of the counterpartys default." They also state (p.24) that "collateral collected as initial margin from the customer is treated as a customer asset" and (p.8) "the collected margin must be subject to arrangements that fully protect the posting party to the extent possible under applicable law in the event that the collecting party enters bankruptcy." Our model is designed to capture these principles in simplified form: collateral belongs to the posting party; it can be seized quickly by the collecting party if the posting party defaults; the return of collateral to the posting party may be delayed by bankruptcy proceedings if the collecting party fails.

In the centrally cleared market, CCPs collect but do not post collateral. Here, too, collateral arrangements ensure immediate access by the CCP in case of a counterpartys default. Trades with a $\mathrm{CCP}$ cannot be unilaterally terminated because the CCP needs to maintain a "matched book," with an offsetting contract for every trade.

\section{References}

[1] Amini, H., Filipovic, D. and Minca, A. (2016). Uniqueness of equilibrium in a payment system with liquidation costs, Operations Research Letters 44(1), 1-5.

[2] Ayer, J.D., Bernstein, M., and Friedland, J. (2004). Chapter 11 - "101," American Bankruptcy Institute Journal 23(8), 16-19.

[3] Banerjee, T., and Feinstein, Z. (2018). Impact of contingent payments on systemic risk in financial networks, Working paper, Department of Electrical and Systems Engineering, Washington University in St. Louis. 
[4] Basel Committee on Banking Supervision and International Organization of Securities Commissions (2015). Margin requirement for non-centrally cleared derivatives, Bank for International Settlements, Basel, Switzerland.

[5] Bichuch, M., and Feinstein, Z. (2019). Optimization of fire sales and borrowing in systemic risk, SIAM Journal on Financial Mathematics 10(1), 68-88.

[6] Bolton, P. and Oehmke, M. (2015). Should derivatives be priviledged in bankruptcy?, Journal of Finance 70, 2353-2394.

[7] Braouezec, Y. and Wagalath, L. (2018). Strategic fire-sales and price-mediated contagion in the banking system, European Journal of Operational Research 274(3), 1180-1197.

[8] Cabrales A., Gale, D., and Gottardi P. (2016) Financial contagion in networks. In The Oxford Handbook of the Economics of Networks, Oxford University Press.

[9] Chang, J.-W. (2018). Collateralized debt networks with lender default, working paper, Department of Economics, Yale University.

[10] Cifuentes, R., Ferrucci, G., and Shin, H.S. (2005). Liquidity risk and contagion, Journal of the European Economic Association 3, 556-566.

[11] Cont, R. and Schaanning, E. (2017) Fire sales, indirect contagion and systemic stress testing, working paper, University of Oxford.

[12] David, A., and Lehar. A. (2017). Imperfect renegotiations in interbank financial networks, Management Science, to appear.

[13] Duffie, D. (2017). Financial regulatory reform after the crisis: an assessment, Management Science 64(10), 4835-4857

[14] Duffie, D., Scheicher, M., and Vuillemey, G. (2015). Central clearing and collateral demand, Journal of Financial Economics 116, 237256.

[15] Duffie, D., and Skeel, D. A. (2012). A dialogue on the costs and benefits of automatic stays for derivatives and repurchase agreements, Penn Law: Legal Scholarship Repository

[16] Duffie, D., and Wang, C. (2017). Efficient contracting in network financial markets, Working paper, Graduate School of Business, Stanford University. 
[17] Eisenberg, L., and Noe, T.H. (2001). Systemic risk in financial systems, Management Science 47, 236-249.

[18] Elsinger, H. (2009). Financial networks, cross holdings, and limited liability, Oesterreichische Nationalbank Working Paper 156.

[19] Federal Deposit Insurance Corporation (2011). The orderly liquidation of Lehman Brothers Holdings Inc. under the Dodd-Frank Act, FDIC Quarterly 5(2), 31-49.

[20] Federal Reserve System (2017). Restrictions on qualified financial contracts of systemically important U.S. banking organizations and the U.S. operations of systemically important foreign banking organizations, Federal Register 82(175), 42882-42926.

[21] Financial Stablity Board (2017). Review of OTC derivatives market reforms, Basel, Switzerland.

[22] Fleming, M.J. and Sarkar, A. (2013). The failure resolution of Lehman Brothers, Economic Policy Review (Dec), 175-206.

[23] Gennaioli, N. and Shleifer, A. (2018). A Crisis of Beliefs: Investor Psychology and Financial Fragility. Princeton University Press.

[24] Ghamami, S., and Glasserman, P. (2017). Does OTC derivatives reform incentivize central clearing?, Journal of Financial Intermediation 32, 76-87.

[25] Glasserman, P. and Young, H.P. (2016). Contagion in financial networks, Journal of Economic Literature 54(3), 779-831.

[26] Ghamami, S. (2020). The unintended impact of swap stays on financial stability, Risk Magazine.

[27] Ghamami, S. (2020). The unintended impact of collateral on financial stability, Risk.

[28] Hurd, T.R. (2016). Contagion! Systemic Risk in Financial Networks, Springer, New York.

[29] ISDA (2019) Legal Derivatives for Smart Derivatives Contracts: The ISDA Master Agreement, International Swaps and Derivatives Association.

[30] Jackson, M. O., and Pernoud, A. (2019) What makes financial markets special? Systemic risk and its measurement in financial networks. Working paper, Department of Economics, Stanford University. 
[31] Jackson, T. (2012). Bankruptcy code chapter 14: a proposal, The Resolution Project, Hoover Institution

[32] Kusnetsov, M., and Veraart, L. A. (2019). Interbank clearing in financial networks with multiple maturities, SIAM Journal on Financial Mathematics 10(1), 37-67.

[33] Milgrom, P., and Roberts, J. (1994). Comparing equilibria, The American Economic Review $84(3), 441-459$.

[34] Roe, M. J., and Adams, S. D. (2015). Restructuring failed financial firms in bankruptcy: selling Lehman's derivatives portfolio, Yale Journal on Regulation 32, 363-411.

[35] Rogers, L. C. G., and Veraart, L. A. (2013). Failure and rescue in an interbank network, Management Science 59(4), 882-898.

[36] Shleifer, A., and Vishny, R. (2011). Fire sales in finance and macroeconomics, Journal of Economic Perspectives 25(1), 29-48.

[37] Sidanius, C., and Zikes, F. (2012). OTC derivatives reform and collateral demand impact, Bank of England Financial Stability Paper 18.

[38] Skeel, D. (2010). The New Financial Deal: Understanding the Dodd-Frank Act and Its (Unintended) Consequences. John Wiley \& Sons.

[39] Skeel, D. A., and Jackson, T. (2012). Transaction consistency and the new finance in bankruptcy. Penn Law: Legal Scholarship Repository.

[40] Stein, J. C. (2013). The fire-sales problem and securities financing transactions. Remarks by Jeremy C. Stein at the Federal Reserve Bank of Chicago and International Monetary Fund Conference, Chicago.

[41] Tarski, A. (1955). A lattice-theoretical fixpoint theorem and its applications, Pacific Journal of Mathematics 5(2), 285-309.

[42] U.S. Department of the Treasury (2017). A financial system that creates economic opportunities: banks and credit unions. Executive Order 13772 on Core Principles for Regulating the United States Financial System.

[43] U.S. Department of the Treasury (2017). A financial system that creates economic opportunities: capital markets. Executive Order 13772 on Core Principles for Regulating the United States Financial System. 
[44] U.S. Department of the Treasury (2018). Orderly Liquidation Authority and Bankrutcy Reform. Executive Order 13772 on Core Principles for Regulating the United States Financial System. 